\title{
A novel method for determining the feasible integral self-stress states for tensegrity structures $^{* *}$
}

https://doi.org/10.1515/cls-2021-0007

Received Sep 15, 2020; accepted Dec 28, 2020

Abstract: The form-finding analysis is a crucial step for determining the stable self-equilibrated states for tensegrity structures, in the absence of external loads. This formfinding problem leads to the evaluation of both the selfstress in the elements and the shape of the tensegrity structure. This paper presents a novel method for determining feasible integral self-stress states for tensegrity structures, that is self-equilibrated states consistent with the unilateral behaviour of the elements, struts in compression and cables in tension, and with the symmetry properties of the structure. In particular, once defined the connectivity between the elements and the nodal coordinates, the feasible self-stress states are determined by suitably investigating the Distributed Static Indeterminacy (DSI). The proposed method allows for obtaining feasible integral self-stress solutions by a unique Singular Value Decomposition (SVD) of the equilibrium matrix, whereas other approaches in the literature require two SVD. Moreover, the proposed approach allows for effectively determining the Force Denstiy matrix, whose properties are strictly related to the super-stability of the tensegrity structures. Three tensegrity structures were studied in order to assess and discuss the efficiency and accuracy of the proposed innovative method.

Keywords: tensegrity structures, self-equilibrium, feasible self-stress states

\footnotetext{
${ }^{\star}$ Corresponding Author: Gaetano Pavone: Department of Civil Engineering and Architecture (DICAR), Polytechnic University of Bari, Bari, Italy; Email: gaetano.pavone@poliba.it Aguinaldo Fraddosio, Mario Daniele Piccioni: Department of Civil Engineering and Architecture (DICAR), Polytechnic University of Bari, Bari, Italy

$\star \star$ Paper included in the Special Issue entitled: Shell and Spatial Structures: Between New Developments and Historical Aspects
}

\section{Introduction}

Tensegrity structures are an intriguing class of reticulated systems and hold promising possibilities in different applications: from architecture $[1,2]$ to civil engineering [36], from biology [7, 8] to aerospace [9-11], as well as from robotics [12-15] to the design of metamaterials [16-20].

Originally proposed by Buckminster Fuller [21], tensegrity structures can be defined as a, usually free-standing, pre-stressed, pin-jointed system, composed by a network of tensile elements (cables) within a discontinuous set of compressed elements (struts). The initial pre-stressed condition allows for the rigidity and the stability of the tensegrity structures [22].

It is evident that the mechanical behaviour of these structures is highly dependent on the self-stress states [23]. Thus a complete analysis of tensegrity structures is made of two key points: first, the form-finding problem, and then the study of the response to the external loads [24].

The process of form-finding depends on the initial input parameters, that is, the geometry of the structure and the level of the self-stress in the elements $[25,26]$. Commonly, both the geometry and the self-stress are unknown variables of the problem. If only the latter is known, i.e. the internal forces in the elements in the self-equilibrium state are defined, the problem reduces to the seeking of the nodal coordinates of the structure, which can be determined from the analysis of the equilibrium states. On the other hand, if the geometry of the tensegrity structure is known, that is, the nodal coordinates and the connectivity between elements are prescribed, the problem turns out to be the initial self-stress identification (force-finding problem) [27].

In the latter case, however, difficulties arise with the evaluation of the level of the self-stress and then of suitable self-stress vectors which taking into account both the unilateral behaviour of the elements and the self-equilibrium of the tensegrity structure [28]. This happens, especially, for tensegrity structures with multiple independent self-stress states [29]. Indeed, in general, the independent self-stress modes obtained from the null-space of the equilibrium ma- 
trix do not meet the predefined unilateral behaviour of the elements [30]. Thus, it is necessary to determine a special combination of such independent self-stress modes in order to define possible feasible self-stress states [31].

It is worth to recall that, a feasible self-stress state is a self-stress state consistent both with the self-equilibrium of the tensegrity structure and the unilateral behaviour of the elements, that is, cables in tension and struts in compression [27]. If a feasible self-stress state also satisfies the symmetry properties of the structure, it takes the name of feasible integral self-stress state [32].

In the recent past, various efficient analytical or numerical form-finding methods [33] have been proposed: among the others, Force Density Method (FDM) [34-36], programming method [37-40], dynamics relaxation method [41, 42], finite-element method [43, 44], optimization-based method [45-47].

In the present work, the FDM has been used in order to tackle the self-equilibrium problem for tensegrity structures.

The concept of the force density, originally proposed in [48], corresponds to the ratio between the internal forces in the elements and their lengths. Such quantities are clearly affected by the sign, i.e. positive for cables and negative for struts. By considering the force densities of the elements, the non-linear problem of the equilibrium can be neatly linearized [49].

Many researchers have made considerable efforts for improving the application of the FDM to the form-finding of tensegrity structures. Among them, Xian et al. [50] proposed an optimization approach based on the FDM and the mixed-integer nonlinear programming for the design of tensegrity structures. The member connectivity, as well as the nodal coordinates and force densities, are simultaneously used as design variables.

Cai et al. [51] studied the form-finding problem of tensegrity structures with multiple equilibrium modes by means of an equivalent optimization problem of an energybased objective function with Lagrange multipliers. Different structural modes corresponding to different symmetry grouping conditions were achieved.

Also, Cai and Feng [52] proposed an efficient formfinding method based on the optimization method; here, the force densities of the elements of a tensegrity structure are obtained by minimizing a special objective function, which satisfies the non-degeneracy necessary condition for the force density matrix.

Zhang and Ohsaki [34] presented a numerical method for the form-finding of tensegrity structures. In particular, eigenvalue analysis and spectral decomposition were carried out iteratively to find the feasible set of force densities that satisfies the requirement on the rank deficiency of the equilibrium matrix with respect to the nodal coordinates.

In addition, Zhang et al. [25] presented a highly efficient form-finding method for tensegrity systems based on the structural stiffness matrix defined as the derivative of the out-of-balance force vector with respect to the nodal coordinate vector.

Lee et al. [53] have studied the truncated polyhedral tensegrity structures by means of a generalized formfinding procedure by using the FDM combined with a genetic algorithm. Additionally, Gan et al. [54] suggested a novel and versatile numerical technique for determining a self-stress state in a combination with a genetic algorithm as a form-finding procedure for an irregular tensegrity structure.

Yuan et al. [55] presented a novel and versatile formfinding method for tensegrity structures based on nonlinear equilibrium equations where the nodal coordinates vectors are variables. The input parameters for the form-finding method are the topology, the initial configuration of the structure, the rest lengths, and the axial stiffness of elements.

Koohestani [56] utilized the Faddeev-LeVerrier algorithm to generate relationships between force densities of elements, providing explicit analytical conditions for selfstressed states. This method only requires sum and multiplications as major computational operations and overcomes complicated triangular factorizations and eigenvalue decompositions of the symbolic force density matrix.

Moreover, Gomez Estrada et al. [57] proposed a numerical form-finding procedure which only requires the specification of the type of each member, i.e. cable or strut, and the connectivity of the nodes. Iterative adjustment of the member forces are made until the state of self-stress is found.

Moreover, for describing the mechanical behaviour of tensegrity structures [58-60], the static and kinematic indeterminacy evaluation can be effectively used as a method for structural identification. For defining the contribution of each element to the total degree of indeterminacy of the structure, also taking into account the influence of the material properties, it can be used the distributed static indeterminacy (DSI) value [61]. Thus, DSI can represent the mechanical behaviour of flexible structures in the primary design. Moreover, in [61] a unified method for the DSI evaluation is proposed, both for kinematically determinate and indeterminate structures. It has been highlighted that since DSI takes into account symmetry properties, a simple but efficient grouping criterion of the elements of the structure can be established for improving the efficiency of the forcefinding method. 
Notice that DSI values are related to both geometric and stiffness symmetry properties of the structure; moreover, stiffness symmetry (depending on the axial stiffness of the elements) can be inconsistent with the geometric symmetry (depending only on the position of the elements).

The application of DSI suggested in [61] concerns the use of DSI as simple and efficient grouping criterion into a specific Force Density Method called Double Singular Value Decomposition (DSVD) [62]. Moreover, DSI values were used as symmetry indicators for generating an initial group classification of the elements of a cable-strut structure for performing a DSVD [63]. However such initial group clustering of elements only reduces the iteration time of seeking a proper grouping scheme for the DSVD.

Moreover, once obtained the self-stress states in the elements, it is possible to determine the Force Density matrix [64], whose characteristics are crucial for studying the self-equilibrium problem and the stability conditions for tensegrity structures $[65,66]$.

Many authors studied different kinds of problems related to the form-finding of the tensegrity structures based on the properties of the Force Density matrix.

Chen et al. [65] pointed out an improved symmetry method for the analytical form-finding of tensegrity structures based on the group representation theory and the FDM. This approach requires only to specify the symmetry properties and the connectivity of the structure. However, with the increase of the element type, the computational complexity of the determination of the Force Density matrix increases.

Based on the characteristic polynomial of the symbolic Force Density matrix, a general analytical scheme for tensegrity form-finding analysis was proposed by Zhang et al. [66]. Also for this case, the proposed method requires high computational efforts as the geometrical complexity of the structure increases

Tran and Lee [67] presented a numerical method for form-finding of tensegrity structures in which the topology and the types of members are the only required information; the eigenvalue decomposition of the Force Density matrix and the single value decomposition of the equilibrium matrix are performed iteratively.

Another relevant issue concerns stability conditions. In this case, the Force Density matrix plays a fundamental role in the analysis of the necessary and sufficient conditions for the super-stability, i.e., the property for a tensegrity structure to be stable irrespectively of the selection of materials and of the level of self-stress in the elements [68]. Indeed, a $d$-dimensional tensegrity structure is said to be super-stable if the Force Density matrix is positive semi- definite and its rank deficiency is equal to $d+1$, and it has a non-degenerate geometry in the $d$-dimensional space [68].

In the literature, to the best of the Author's knowledge, the force-finding problem for tensegrity with multiple independent self-stress modes has been carried out by using cumbersome approaches: optimization techniques, mixedinteger nonlinear programming strategies, spectral decompositions, stiffness matrix evaluations and numerical iterative procedures.

Thus, as mentioned above, a more efficient algorithm for determining the feasible integral self-stress states for tensegrity structures by using the DSI values needs to be investigated. In particular, it should be avoided the second Singular Value Decomposition (SVD) for reducing timeconsuming inherent the grouping operation.

In this paper, an innovative and efficient method for determining feasible integral self-stress states for tensegrity structures is proposed by considering the Distributed Static Indeterminacy (DSI) evaluation. The only required initial data are the topology of the structure, i.e. the connectivity relations between the elements and their types (cables or struts), and the nodal coordinates.

Two advantages of the proposed approach can be remarked. First, a unique (SVD) of the equilibrium matrix has to be carried out for determining the independent selfstress modes, which span the null-space of this matrix, then through the DSI evaluation it is possible to determine the feasible self-stress states. To this aim, a linear combination of the independent self-stress modes consistent with the flexibility properties of the elements of the tensegrity structure can be evaluated. From this stems the second advantage consisting in the possibility of obtaining different feasible self-stress states according to the design needs by choosing the material parameters of the elements, that is the Young's modulus and the cross-sectional area.

Such innovative method can be especially useful for the analysis of tensegrity structures with multiple independent self-stress states. Unlike the existing methods in literature $[27,69,70]$, in the proposed approach the combined conditions coming from the stiffness symmetry and the geometry symmetry of the tensegrity structure can be satisfied without using further grouping operations, which usually are inferred from a visual inspection of the structure.

Furthermore, it can be noted that the Force Density matrix is strictly related to the connectivity properties of the system, i.e. the relations between the elements of the structure and the nodes, and to the level of the self-stress in the elements.

The approach here proposed effectively allows for determining the Force Density matrix and its properties with 
a low computational cost. It reveals to be useful for all the analysis for the tensegrity structures above recalled: the form-finding analysis, the investigation of the superstability conditions, and the study of the relations between elements and of the self-stress level according to the actual axial stiffness of the elements.

The paper is organized as follows: Section 2 briefly introduces the basic idea of the FDM. In Section 3, the concept of the DSI is recalled and its application to the tensegrity structures is explained. Section 4 is devoted to the descrip tion of the novel method here proposed. Section 5 recalls the definition of the Force Density matrix and illustrates its formulation according to the proposed approach. Finally, for validating the method several well-known tensegrity structures are studied in Section 6.

\section{Force Density Method}

In this Section, we briefly recall the self-equilibrium problem for tensegrity structures. The following assumptions are made:

- elements (struts and cables) are rectilinear and connected only at their ends by pin-joints;

- nodal coordinates and nodal connectivity are given;

- no external loads are applied;

- the cross-sectional area $A$ of each element remains unchanged under the pre-stress.

We consider a tensegrity structure with $e$ elements $\left(s_{t}\right.$ struts and $c$ cables, that is, $s_{t}+c=e$ ) connected to $n$ nodes $(e<3 n)$. Nodal coordinates are expressed in a Cartesian orthogonal reference system $O\left\{\mathbf{e}_{\mathrm{x}}, \mathbf{e}_{\mathrm{y}}, \mathbf{e}_{\mathrm{z}}\right\}$ and are collected in three vectors $\mathbf{x}, \mathbf{y}$ and $z \in \mathbb{R}^{n}$, respectively.

By the Graph Theory [71], member connectivity relations can be expressed by means of the so-called Connectivity matrix $\mathbf{C} \in \mathbb{R}^{\text {exn }}$ [36]. In particular, if the member $k$ connects the node $i$ to the node $j$, then the $k$-th row of $\mathbf{C}$ has only two non-zero entries in the $i$-th and $j$-th position $(i<j)$, which are equal to 1 and -1 respectively. Hence:

$$
\begin{aligned}
{\left[\mathbf{C}_{k, p}\right.} & = \begin{cases}+1 & \text { if } p=i \\
-1 & \text { if } p=j \\
0 & \text { otherwise }\end{cases} \\
k & =1, \ldots, e, p=1, \ldots, n .
\end{aligned}
$$

Furthermore, the length $l_{k}$ of the $k$-th member of the structure can be expressed as:

$$
l_{k}=\sqrt{\left(x_{i}-x_{j}\right)^{2}+\left(y_{i}-y_{j}\right)^{2}+\left(z_{i}-z_{j}\right)^{2}} .
$$

For our purposes, the matrix $\mathbf{L} \in \mathbb{R}^{e x e}$ is defined as the diagonal matrix by collecting the lengths of the elements.

The self-equilibrium problem can be solved by using FDM. To this aim, for the $k$-th element of the structure it is possible to define the force density $q_{k}$ :

$$
q_{k}=\frac{t_{k}}{l_{k}}
$$

where $t_{k}$ denotes the internal force in the element $k\left(t_{k}\right.$ is positive for cables and negative for struts) in the selfstress state. Force densities of the elements can be grouped in the vector $\mathbf{q} \in \mathbb{R}^{e}=\left\{q_{1}, q_{2}, \ldots, q_{k}\right\}$, whose matrix diagonalization is $\mathbf{Q} \in \mathbb{R}^{\text {exe }}$, i.e., $\mathbf{Q}=\operatorname{diag}(\mathbf{q})$.

Considering both Eq. (1) and Eq. (3), the equilibrium equations for the tensegrity structure in the three directions $\mathbf{e}_{\mathrm{x}}, \mathbf{e}_{\mathrm{y}}$, and $\mathbf{e}_{\mathrm{z}}$ can be then expressed in the following matrix linear form [33]:

$$
\left\{\begin{array}{l}
\mathbf{C}^{\mathrm{T}} \mathbf{Q} \mathbf{C x}=\mathbf{0} \\
\mathbf{C}^{\mathrm{T}} \mathbf{Q} \mathbf{C y}=\mathbf{0}, \\
\mathbf{C}^{\mathrm{T}} \mathbf{Q} \mathbf{C z}=\mathbf{0}
\end{array}\right.
$$

where the superscript " $\mathrm{T}$ " indicates the usual matrix transposition operation.

Alternatively, by considering the element internal forces vector $\mathbf{t} \in \mathbb{R}^{e}=\left\{t_{1}, t_{2}, \ldots, t_{k}\right\}$, the equilibrium equations in Eq. (4) can be written as:

$$
\left\{\begin{array}{l}
\mathbf{C}^{\mathrm{T}} \operatorname{diag}(\mathbf{C x}) \mathbf{L}^{-1} \mathbf{t}=\mathbf{0} \\
\mathbf{C}^{\mathrm{T}} \operatorname{diag}(\mathbf{C y}) \mathbf{L}^{-1} \mathbf{t}=\mathbf{0} \\
\mathbf{C}^{\mathrm{T}} \operatorname{diag}(\mathbf{C z}) \mathbf{L}^{-1} \mathbf{t}=\mathbf{0}
\end{array}\right.
$$

By introducing the equilibrium matrix $\mathbf{A} \in \mathbb{R}^{3 n x e}$ [64], Eq. (5) can be rewritten in a compact form as:

$$
\text { At }=\mathbf{0},
$$

where the equilibrium matrix A can be expressed as:

$$
\mathbf{A}=\left[\begin{array}{l}
\mathbf{C}^{\mathrm{T}} \operatorname{diag}(\mathbf{C x}) \mathbf{L}^{-1} \\
\mathbf{C}^{\mathrm{T}} \operatorname{diag}(\mathbf{C y}) \mathbf{L}^{-1} \\
\mathbf{C}^{\mathrm{T}} \operatorname{diag}(\mathbf{C z}) \mathbf{L}^{-1}
\end{array}\right] .
$$

Let $r_{A}$ be the rank of $\mathbf{A}$; if $r_{A}<e$, non-trivial solutions exist. These non-trivial solutions correspond to $s$ independent self-stress modes, which can be viewed as the bases of the vector space of the internal forces in the elements, with:

$$
s=e-r_{A} \geq 1 .
$$

Hence, it is possible to define a matrix $\mathbf{S} \in \mathbb{R}^{\text {exs }}$ whose $i$-th column is the $\mathbf{s}_{i}$ independent self-stress mode, i.e.:

$$
\mathbf{S}=\left[\mathbf{s}_{1}, \mathbf{s}_{2}, \ldots, \mathbf{s}_{s}\right]=\left[\begin{array}{cccc}
s_{11} & s_{21} & \cdots & s_{s 1} \\
s_{12} & s_{22} & \cdots & s_{s 2} \\
\vdots & \vdots & \cdots & \vdots \\
s_{1 e} & s_{2 e} & \cdots & s_{s e}
\end{array}\right] .
$$


A general solution of Eq. (6) can be determined as a linear combination of $s$ independent self-stress modes [29], that is:

$$
\mathbf{t}=\mathbf{S} \boldsymbol{\alpha},
$$

where $\alpha_{i}, i=1,2, \ldots, s$, are arbitrary real coefficients of the linear combination collected in the vector $\alpha \in \mathbb{R}^{s}$.

If the null-space of the equilibrium matrix $\mathbf{A}$ is spanned by a unique independent self-stress mode, i.e. if $s=1$, then such vector represents the only feasible self-stress state of the structure. In this case, the matrix $\mathbf{S}$ becomes a column vector. It can be noted that in this case, the independent self-stress mode should be consistent with the unilateral behaviour of the elements for determining the feasible selfstress states.

If there are multiple independent self-stress modes, i.e. if $s>1$, then it is necessary to calculate suitable linear combinations of these bases by means of Eq. (10) since such modes, usually, do not satisfy the unilateral behaviour of the elements as well as the symmetry of the structure.

Indeed, independent self-stress modes resulting from the null-space of the equilibrium matrix usually only satisfy the nodal equilibrium conditions, thus cannot be utilized directly. On the other hand, unilateral conditions related to the mechanical behaviour of struts and cables are not considered in the formulation of the matrix $\mathbf{A}$.

However, for statically indeterminate structures $(s>1)$ exhibiting symmetry properties, as is often the case for tensegrity structures, many elements can be collected into suitable groups according to the symmetry [28]. In this vein, the evaluation of Eq. (10) can be simplified taking into account the symmetry constraints of the geometry of the structure, that is, the same self-stress can be assigned to elements in the same symmetric position. Thus, it can be viewed as a constraint on the self-stress distribution in the elements of the structure.

Definitively, the aim is the evaluation of the self-stress distribution in the elements consistent with the symmetry properties of the structure and their unilateral behaviour, that is the feasible integral self-stress states.

\section{Distributed static indeterminacy}

Let $\mathbf{d} \in \mathbb{R}^{3 n}$, and $\mathbf{e} \in \mathbb{R}^{e}$ denote the vector of infinitesimal nodal displacements and the vector of member elongations, respectively. It is possible to define the relations among such kinematic variables in terms of the compatibility matrix $\mathbf{B} \in \mathbb{R}^{e x 3 n}[60]$ such that:

$$
\mathbf{B d}=\mathbf{e} .
$$

From the principle of virtual work, it follows that $\mathbf{B}=\mathbf{A}^{\mathrm{T}}$ [58]. Let $r_{B}$ be the rank of $\mathbf{B}\left(r_{B}=r_{A}\right)$; then the number $m$ of the possible mechanisms which span the null-space of $\mathbf{B}$ is $m=3 n-r_{B}$. Moreover, the number $m_{i}$ of the infinitesimal mechanisms can be obtained by excluding the rigid-body motions in the three-dimensional space, i.e., $m_{i}=m-6$.

Taking into account the effects of initial elongations $e_{k}$, $k=1,2, \ldots, e$, under the pre-stress, and by assembling the initial elongations vector $\mathbf{e}_{0} \in \mathbb{R}^{e}$, constitutive equations for the tensegrity structures can be then expressed as [61]:

$$
\mathbf{e}=\mathbf{e}_{0}+\mathbf{F t},
$$

where $\mathbf{F} \in \mathbb{R}^{\text {exe }}$ is the diagonal flexibility matrix, whose $k$-th diagonal entry is $l_{k} / E_{k} A_{k}$, with $E_{k}$ and $A_{k}$ the Young's modulus and the cross-sectional area of the element, respectively.

Moreover, in the standard linear algebraic theory of vector spaces, it results that all the information required for the analysis of a framework are contained in the four fundamental vector spaces associated with the equilibrium matrix $\mathbf{A}$ (for further details about their kinematic and static interpretation [59]).

In particular, the row-space, the null-space, the column-space and the left null-space of $\mathbf{A}$, can be associated with the equilibrium matrix. In particular, the left null-space and the null-space of $\mathbf{A}$ are spanned by the $m_{i}$ infinitesimal mechanisms and the $s$ independent self-stress modes, respectively. For what considered below, it is possible to recall the well-known properties of orthogonality among such vector subspaces [59], thus it is possible to write:

$$
\mathbf{S}^{\mathrm{T}}\left(\mathbf{e}_{0}+\mathbf{F t}\right)=\mathbf{0},
$$

and substituting Eq. (9) in (13):

$$
\mathbf{S}^{\mathrm{T}}\left(\mathbf{e}_{0}+\mathbf{F S} \boldsymbol{\alpha}\right)=\mathbf{0} .
$$

It is possible to recall that for a full rank matrix $\mathbf{P} \in \mathbb{R}^{i x j}$, with $\mathbf{j} \leq \mathrm{i}$, the square matrix $\mathbf{P}^{\mathrm{T}} \mathbf{P}$ is always positive definite. Moreover, let $\mathbf{Q} \in \mathbb{R}^{i x i}$ symmetric and positive definite, then $\mathbf{P}^{\mathrm{T}} \mathbf{Q P}$ is a symmetric, non-singular, positive definite matrix. Thus, the matrix $\mathbf{S}^{\mathrm{T}} \mathbf{F S}$ is a symmetric, non-singular, positive definite matrix.

Therefore, from Eq. (14) it is possible to determine the vector $\boldsymbol{\alpha}$ as:

$$
\boldsymbol{\alpha}=-\left(\mathbf{S}^{\mathrm{T}} \mathbf{F S}\right)^{-1} \mathbf{S}^{\mathrm{T}} \mathbf{e}_{0} .
$$

Hence, the element internal forces vector $\mathbf{t}$ can be obtained by substituting Eq. (15) into Eq. (10):

$$
\mathbf{t}=-\mathbf{S}\left(\mathbf{S}^{\mathrm{T}} \mathbf{F S}\right)^{-1} \mathbf{S}^{\mathrm{T}} \mathbf{e}_{0}
$$


By introducing the diagonal stiffness matrix $\mathbf{K} \in \mathbb{R}^{\text {exe }}$, such that $\mathbf{K}=\mathbf{F}^{-1}$, (the $k$-th diagonal entry of $\mathbf{K}$ is $\mathrm{E}_{k} \mathrm{~A}_{k} / l_{k}$ ) Eq. (15) can be rewritten as:

$$
\mathbf{t}=-\mathbf{K}\left[\mathbf{F S}\left(\mathbf{S}^{\mathrm{T}} \mathbf{F S}\right)^{-1} \mathbf{S}^{\mathrm{T}}\right] \mathbf{e}_{0}=-\mathbf{K} \mathbf{\Omega} \mathbf{e}_{0},
$$

where the square matrix $\mathbf{\Omega} \in \mathbb{R}^{\text {exe }}\left(=\mathbf{F S}\left(\mathbf{S}^{\mathrm{T}} \mathbf{F S}\right)^{-1} \mathbf{S}^{\mathrm{T}}\right)$ correlates different aspects of the structure: the geometrical configuration, the topology and the stiffness properties of the elements, defined by the designers. Since the matrix $\mathbf{S}^{\mathrm{T}} \mathbf{F S}$ in Eq. (15) is always positive definite; Eq. (17) is applicable for both kinematically determinate and indeterminate structures. Equation (17) is a constitutive equation describing the relation between the internal forces in the elements and their initial elongation. From the definition of the square matrix $\mathbf{\Omega}$, it results that $\mathbf{\Omega}$ is an idempotent singular matrix, that is, $\boldsymbol{\Omega}^{2}$ is equal to $\boldsymbol{\Omega}$, hence its eigenvalues are either 0 or 1 . Furthermore, the rank of $\mathbf{\Omega}$ is equal to the sum of its eigenvalues, or equivalently, is equal to its trace. The sum of all the main diagonal elements $\gamma_{i}(i=1,2, \ldots, e)$ is equal, thus, to the total degree $s$ of static indeterminacy of the structure. Such diagonal entries $\gamma_{i}$, collected into the vector $\boldsymbol{\omega} \in \mathbb{R}^{e}$, are defined in the literature as Distributed Static Indeterminacies (DSI) [63]: indeed $\gamma_{i}$ represents the contribution of the $i$-th element of the structure to its total degree of static indeterminacy.

Moreover, it is possible to show that elements having the same symmetry properties have the same DSI values; indeed, DSI can be viewed as an indicator of the symmetry properties of the structure [61].

Finally, if the flexibility matrix $\mathbf{F}$ is equal to the identity matrix I, then the matrix $\mathbf{\Omega}$ becomes the matrix $\mathbf{\Omega}_{\mathrm{m}}=$ $\mathbf{S}\left(\mathbf{S}^{\mathrm{T}} \mathbf{S}\right)^{-1} \mathbf{S}^{\mathrm{T}} \in \mathbb{R}^{\text {exe }}$, whose diagonal terms can be collected in the vector $\boldsymbol{\omega}_{\mathrm{m}} \in \mathbb{R}^{e}$. It can be noted that the matrix $\boldsymbol{\Omega}_{\mathrm{m}}$, in addition to the above-recalled algebraic properties of the matrix $\Omega$, is characterized by the further property of being always symmetric. In this particular case, the matrix $\boldsymbol{\Omega}_{\mathrm{m}}$ is not affected by the axial stiffness properties of the elements; hence, it is strictly related to the self-equilibrium conditions of the structure.

\section{The new approach for the determination of the feasible integral self-stress states}

We consider a test vector $\mathbf{t}_{\mathrm{p}} \in \mathbb{R}^{e}$, consistent with the sign of the internal forces in the elements, i.e. positive in the cables and negative in the struts, built as follows:

$$
\mathbf{t}_{\mathrm{p}, i}=\left\{\begin{array}{ll}
+1 & \text { if element } i \text { is a cable } \\
-1 & \text { if element } i \text { is a strut }
\end{array}, \quad i=1, \ldots, e .\right.
$$

By considering a single element of the structure subjected to an initial elongation, it results that shortening generates tension, while extension creates compression. Thus, an initial elongations vector $e_{0}$ can be associated with the test vector $t_{p}$ :

$$
\mathbf{e}_{0}=-\mathbf{F} \mathbf{t}_{\mathrm{p}}
$$

Substituting Eq. (19) into Eq. (17), we have:

$$
\mathbf{t}=\mathbf{K} \mathbf{\Omega} \mathbf{F} \mathbf{t}_{\mathrm{p}} .
$$

It is easy to prove that $\mathbf{K} \mathbf{\Omega} \mathbf{F}$ is equal to $\mathbf{\Omega}^{\mathrm{T}}$ (see Appendix A); therefore, Eq. (20) can be rearranged as:

$$
\mathbf{t}_{\mathrm{n}}=\mathbf{\Omega}^{\mathrm{T}} \mathbf{t}_{\mathrm{p}} .
$$

From the definition of the matrix $\mathbf{\Omega}$ and from Eq. (18), the internal forces vector $\mathbf{t}_{\mathrm{n}}$ obtained from the Eq. (21) takes into account both the unilateral behaviour of the elements and the self-equilibrium conditions of the structure. Moreover, as it results from the numerical experiments performed in Section 6, symmetric assignments of the axial stiffness of the elements lead to a symmetric distribution of the internal forces in the elements. Thus, such a vector represents a feasible integral self-stress vector for the tensegrity structure. Moreover, it is worth to observe that, since the definition of the matrix $\mathbf{\Omega}$, the Eq. (21) is strictly related to the material properties of the elements, represented by the matrix $\mathbf{F}$.

In order to verify the accuracy of the numerical analyses performed in Section 6, the vector $\boldsymbol{\varepsilon}_{u} \in \mathbb{R}^{e}$ represents the unbalanced residual normalized internal forces defined as:

$$
\boldsymbol{\varepsilon}_{u}=\mathbf{A t},
$$

and its Euclidean norm can be used to evaluate the numerical errors.

The proposed method, coded using Wolfram Mathematica 11.0, can be outlined as follows. Assigned the element connectivity, by means of the matrix $\mathbf{C}$, and the geometry of the structure in terms of the nodal coordinate vectors $\mathbf{x}$, $\mathbf{y}$ and $\mathbf{z}$, then:

Step 1: Assemble the equilibrium matrix $\mathbf{A}$ by using Eq. (7).

Step 2: Define the material parameters of the elements, that is, the Young's modulus $E_{k}$ and the crosssectional area $A_{k}$, and then construct the flexibility matrix $\mathbf{F}$. 
Step 3: Collect the prototype vector $t_{\mathrm{p}}$, according to the unilateral behaviour of the elements, by means of Eq. (18).

Step 4: Determine the null-space of the equilibrium matrix $\mathbf{A}$ and then assemble the self-stress matrix $\mathbf{S}$.

Step 5: Calculate the matrix $\mathbf{\Omega}$ and thereafter evaluate the feasible self-stress states $\mathbf{t}_{\mathrm{n}}$ by using the Eq. (21).

Step 6: Compute the norm of the unbalanced residual normalized internal forces vectors in order to verify the accuracy of the analyses.

It is worth to note that for tensegrity structures with a unique independent self-stress mode, that is $s=1$, the feasible self-stress states calculated by using the Eq. (21) is obviously not affected by the assignments of the axial stiffness of the elements. Conversely, for tensegrity structures with multiple independent self-stress modes, that is, for $s>1$, different choices of the axial stiffness of the elements lead to different linear combinations of the above-mentioned independent self-stress modes, hence, to different self-stress states.

Conclusively, it can be noted that symmetric distributions of the axial stiffness of the elements correspond to symmetric internal forces in the elements, thus lead to feasible integral self-stress states consistent both with the stiffness symmetry and the geometrical symmetry properties of the structure.

\section{An efficient approach for determining the Force Density Matrix}

In this section, we briefly recall the definition of the Force Density matrix [64], always a square symmetric matrix. In particular, by using the Eq. (4), the equilibrium equations for the tensegrity structure, projected in the three directions $\mathbf{e}_{\mathrm{x}}, \mathbf{e}_{\mathrm{y}}$, and $\mathbf{e}_{\mathrm{z}}$, can be then expressed in the following matrix linear form:

$$
\left\{\begin{array}{l}
\mathbf{D}_{\mathrm{S}} \mathbf{x}=\mathbf{0} \\
\mathbf{D}_{\mathrm{S}} \mathbf{y}=\mathbf{0}, \\
\mathbf{D}_{\mathrm{S}} \mathbf{Z}=\mathbf{0}
\end{array}\right.
$$

with $\mathbf{D}_{\mathrm{s}} \in \mathbb{R}^{\text {exe }}$ the Force Density matrix, defined as follows:

$$
\mathbf{D}_{\mathrm{S}}=\mathbf{C}^{\mathrm{T}} \mathbf{Q} \mathbf{C} \text {. }
$$

A non-degenerate tensegrity structure is super-stable, if the rank deficiency $n_{D}$ of the Force Density matrix, that is the number of its null eigenvalues, is equal to $d+1\left(\lambda_{1}=\right.$ $\ldots=\lambda_{d+1}=0$ ), and the remaining eigenvalues are strictly positive $\left(0<\lambda_{d+2} \leq \ldots \leq \lambda_{e}\right)$.

Eq. (24) represents the standard formulation of the Force Density matrix $\mathbf{D}_{\mathrm{s}}$. The major difficulties in evaluating $\mathbf{D}_{\mathrm{s}}$ trough Eq. (24) comes from the determination of the components of the diagonal matrix $\mathbf{Q}$, especially for tensegrity structures with multiple independent self-stress states, as explained in the previous section.

We recall that independent self-stress modes, usually, are not consistent with the signs of the internal forces in the elements, i.e. positive for cables and negative for struts, since the self-equilibrium conditions do not take into account the unilateral behaviour of the elements. Moreover, it can be observed that for a tensegrity structure with multiple independent self-stress states the Force Density matrix obtained by considering a single independent self-stress mode should be indefinite, that is, it is neither positive semi-definite nor negative semi-definite.

For these reasons, here an alternative formulation of the Force Density matrix $\mathbf{D}_{\mathrm{s}}$ is proposed. Indeed, by recalling the algorithm for the determination of the feasible integral self-stress states proposed in the previous section, and by using the Eq. (21) and Eq. (3), it is possible to rewrite $\mathbf{D}_{\mathrm{s}}$ as follows:

$$
\begin{aligned}
\mathbf{D}_{\mathrm{s}} & =\mathbf{C}^{\mathrm{T}} \mathbf{Q} \mathbf{C}=\mathbf{C}^{\mathrm{T}} \operatorname{diag}\left(\mathbf{L}^{-1} \mathbf{t}_{\mathrm{n}}\right) \mathbf{C} \\
& =\mathbf{C}^{\mathrm{T}} \operatorname{diag}\left(\mathbf{L}^{-1} \mathbf{\Omega}^{\mathrm{T}} \mathbf{t}_{\mathrm{p}}\right) \mathbf{C},
\end{aligned}
$$

where it is recalled that the diagonal matrix $\mathbf{Q}$ is equal to $\operatorname{diag}\left(\mathbf{L}^{-1} \mathbf{t}_{\mathrm{n}}\right)$.

Since our approach allows for efficiently evaluating the feasible integral self-stress states of a tensegrity structure (see Sect. 4), it can now effectively employed also for calculating the Force Density matrix that can be determined by performing a unique SVD of the equilibrium matrix of the structure and by evaluating the DSI values of the elements.

Notice that, different choices of the feasible self-stress states lead to different Force Density matrices and, thus, to different eigenvalues.

Since the geometry and the connectivity properties of the structure are given, the equilibrium matrix A, see Eq. (7), remains unchanged, that is, such matrix is constant in the force-finding problem in the Eq. (6).

Thus, the force-density problem in the Eq. (23) can be seen as an alternative representation of the self-equilibrium problem represented by Eq. (7).

Hence, rank deficiency, as well as, the sign of non-zero eigenvalues of the Force Density matrix, evaluated by using Eq. (25), remain unvaried irrespective of any feasible selfstress state considered. 
Hence, by using the Eq. (25) it is possible to effectively evaluate the matrix $\mathbf{D}_{\mathrm{s}}$ also for verifying the super-stability conditions for tensegrity structures.

\section{Numerical examples}

In this section, three well-known tensegrity structures have been studied in order to compare the results available in the literature with the results obtained by means of the new method here proposed. In particular, we analyze the following tensegrity structures: the Quadruplex; the Snelson's X beam with three modules; the Octahedral cell.

Different assignments of the Young's modulus and the cross-sectional area of the elements were made in order to calculate the corresponding different feasible self-stress states by evaluating the DSI vector $\boldsymbol{\omega}$ and the corresponding internal force vector $\mathbf{t}$.

Specifically, for each of the three tensegrity structures, the analysis was conducted by considering five different conditions:

1) the case in which the flexibility matrix is equal to the identity matrix, that is, $\mathbf{F}=\mathbf{I}$; in this case, the DSI vector coincides with $\boldsymbol{\omega}_{\mathrm{m}}$ and the corresponding internal force vector is denoted by $\mathbf{t}_{\mathrm{nm}}$;

2) a possible assignment of the axial stiffness of the elements which lead to the results reported by the literature; in such case, the vector named $\boldsymbol{\omega}$ (literature) and the vector termed $\mathbf{t}$ (literature) were calculated

3), 4) and 5) two symmetric distribution (called $\mathrm{n} 1$ and n2) of the axial stiffness of the elements and a not-symmetric distribution (called n3) of the axial stiffness of the elements, which yield to the determination of the DSI vectors $\boldsymbol{\omega}_{\mathrm{n} 1}, \boldsymbol{\omega}_{\mathrm{n} 2}$, $\boldsymbol{\omega}_{\mathrm{n} 3}$ and of the related internal force vectors $\mathbf{t}_{\mathrm{n} 1}, \mathbf{t}_{\mathrm{n} 2}, \mathbf{t}_{\mathrm{n} 3}$, respectively.
Furthermore, for both the Snelson's X beam with three modules and the Octahedral cell (tensegrity structures with multiple independent self-stress modes) three further distributions of the axial stiffness of the elements, which also allow for determining results equal to those reported by the literature, have been considered.

Moreover, for the tensegrity structures analysed, the Force Density matrices have been calculated. Their rank deficiencies, as well as, their eigenvalues have been determined in order to evaluate the super-stability conditions of the structures.

Finally, in order to compare the results corresponding to different stiffness properties, the internal force vectors have been normalized, and the force densities of the elements have been normalized respect to the force density of the elements belonging to the first group.

\subsection{Quadruplex}

The tensegrity Quadruplex analyzed, see Figure 1, consists of $n=8$ nodes and $e=16$ elements, i.e. 4 struts and 12 cables,
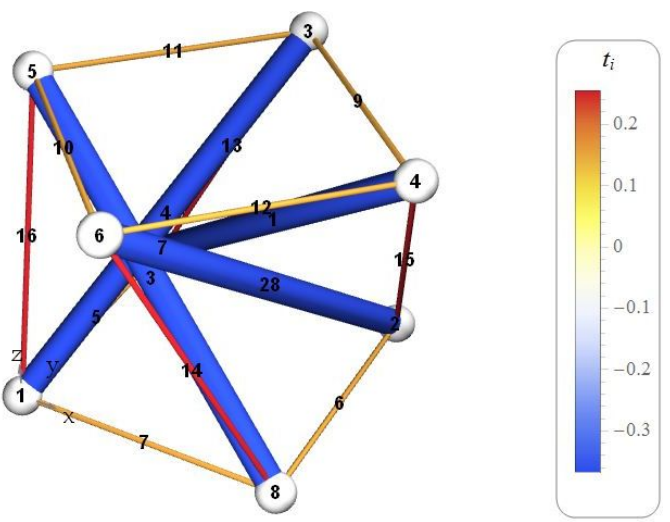

Figure 1: Quadruplex, perspective view. Thick cylinders represent the struts. Different colours have been assigned according to the value of the internal forces in the elements, which are labelled according to the connectivity matrix

Table 1: Axial stiffness of the elements of the Quadruplex

\begin{tabular}{ccccccc}
\hline & $\mathbf{F}=\mathbf{I}$ & literature & $\mathrm{n} 1$ & $\mathrm{n} 2$ & $\mathrm{n} 3$ & \\
Element & $E_{k} A_{k}(\mathrm{~N})$ & $E_{k} A_{k}(\mathrm{~N})$ & $E_{k} A_{k}(\mathrm{~N})$ & $E_{k} A_{k}(\mathrm{~N})$ & $E_{k} A_{k}(\mathrm{~N})$ & Element \\
\hline struts (1-4) & 1645.33 & $10^{6}$ & $10^{6}$ & $10^{6}$ & $10^{6}$ & struts (1-3) \\
cables (5-12) & 1000 & $49 \cdot 10^{3}$ & $49 \cdot 10^{3}$ & $24.5 \cdot 10^{3}$ & $1.5 \cdot 10^{6}$ & strut (4) \\
cables (13-16) & 1137.05 & $49 \cdot 10^{3}$ & $24.5 \cdot 10^{3}$ & $49 \cdot 10^{3}$ & $24.5 \cdot 10^{3}$ & cables (5-10) \\
& & & & & $49 \cdot 10^{3}$ & cables (11-12) \\
& & & & & $49 \cdot 10^{3}$ & cables (13-15) \\
& & & & & $39.2 \cdot 10^{3}$ & cable (16) \\
\hline
\end{tabular}


and its geometrical configuration can be found in [61]. In particular, the top square base and the bottom square base are rotated with respect to each other by a twist angle equal to $\pi / 4$; such bases are inscribed in a circle of radius equal to $707 \mathrm{~mm}$, the height of the prism is equal to $1000 \mathrm{~mm}$. In Table 1 are shown the assignments of the axial stiffness of the elements of the Quadruplex, whereas the corresponding DSI values of the elements are shown in Figure 2.

As it can be observed, by increasing the axial stiffness of an element of the structure, its DSI value decreases whereas the DSI values of the other elements increase. Moreover, symmetric assignments of the axial stiffness, namely the first four cases analysed, lead to a symmetric distribu- tion of DSI values. The rank of the equilibrium matrix $\mathbf{A}$ is 15 , thus the structure has one self-stress mode, i.e. $s=1$, and it possesses 3 infinitesimal mechanisms. Furthermore, by using Eq. (21), it is possible to evaluate the feasible selfstress states, see Figure 3. The figure clearly shows that, as expected, the normalized internal force vectors do not change as the axial stiffness's of the elements vary, also for not-symmetric distribution of the axial stiffness.

As it can be noted in Figure 3, the elements of the Quadruplex can be collected in three groups according to their internal forces, as well as to their force densities: struts (1-4), cables (5-12) and cables (13-16). In particular, the normalized force density of the elements of the first group is

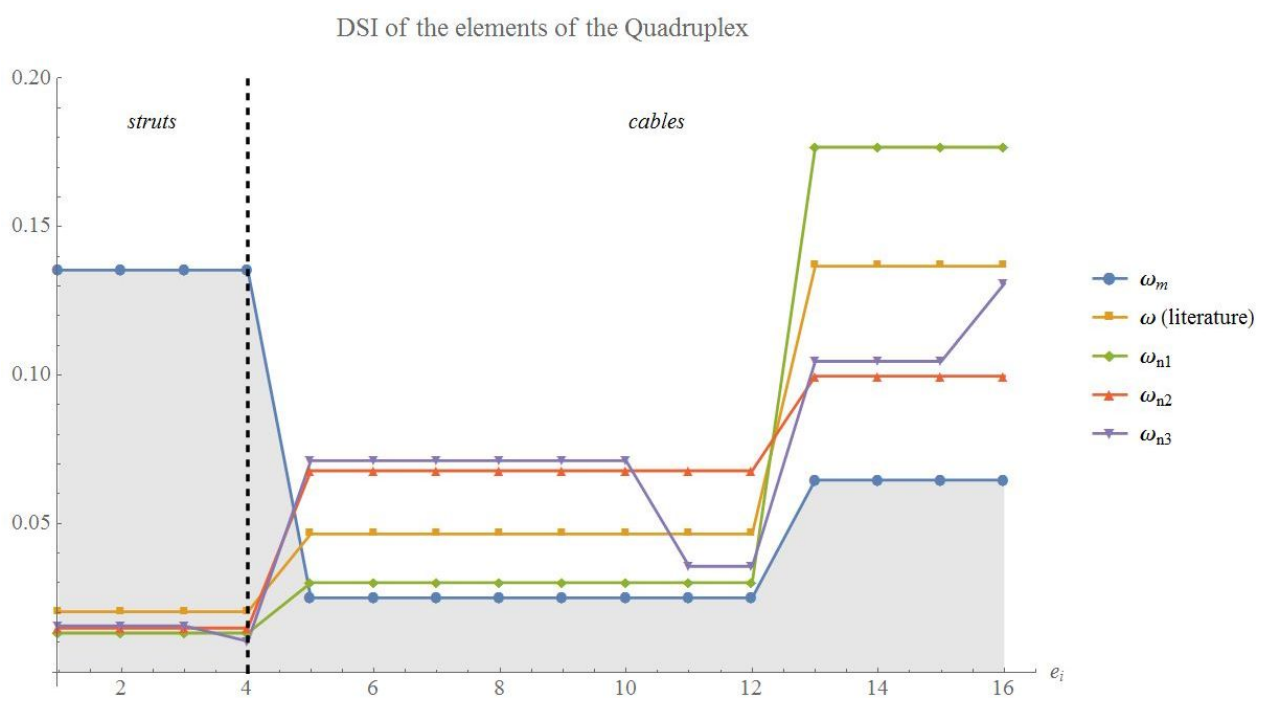

Figure 2: DSI of the elements of the Quadruplex for different assignments of the axial stiffness

Internal forces in the elements of the Quadruplex

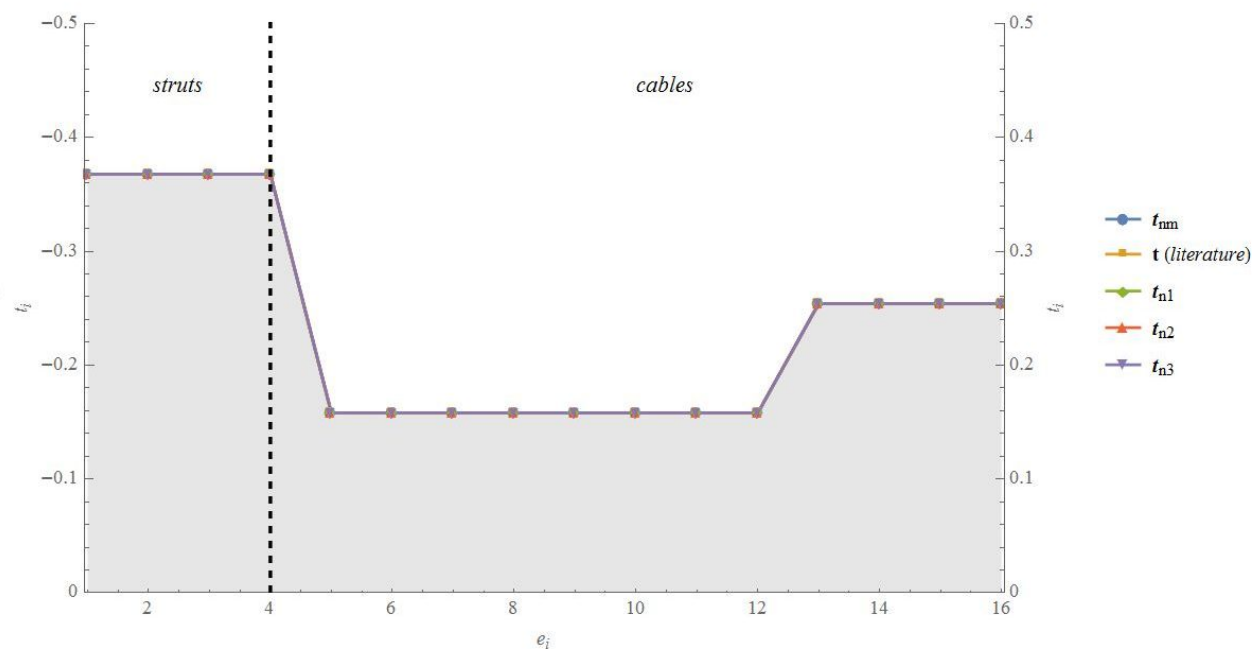

Figure 3: Internal forces in the elements of the Quadruplex as the axial stiffness of the elements vary 
Eigenvalues of the Force Density matrix of the Quadruplex

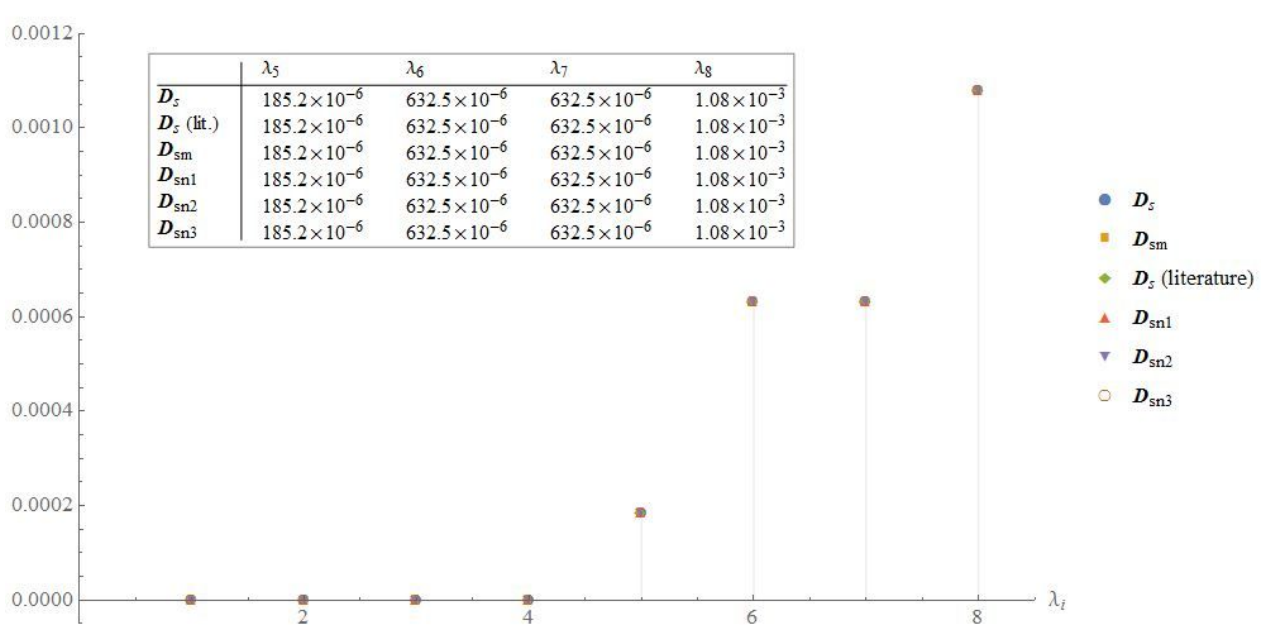

Figure 4: Eigenvalues of the Force Density matrices of the Quadruplex

Table 2: Norm of the unbalanced residual normalized internal forces vectors of the Quadruplex

\begin{tabular}{lccccc}
\hline & $\mathbf{t}_{\mathrm{nm}}$ & $\mathbf{t}$ (literature) & $\mathbf{t}_{\mathrm{n} 1}$ & $\mathbf{t}_{\mathrm{n} 2}$ & $\mathbf{t}_{\mathrm{n} 3}$ \\
\hline$\left[\left[\boldsymbol{\varepsilon}_{u}\right]\right]$ & $5.54 \cdot 10^{-16}$ & $5.73 \cdot 10^{-16}$ & $5.76 \cdot 10^{-16}$ & $6.03 \cdot 10^{-16}$ & $5.82 \cdot 10^{-16}$ \\
\hline
\end{tabular}

equal to -1 , the normalized force density of the elements of the second group is equal to about 0.7071 , and the normalized force density of the elements of the third group is equal to 1.

Moreover, from the analysis of the Force Density matrix, it results that the Quadruplex is a super-stable tensegrity structure. Indeed, its Force Density matrix is a positive semi-definite matrix with four zero eigenvalues, as shown in Figure 4. Obviously, the corresponding eigenvalues evaluated in the five different assignments of the axial stiffness of the elements are identical.

Finally, the norms of the unbalanced residual normalized internal forces vectors are calculated in order to verify the accuracy of the method. As it is shown in Table 2, such norms are close to zero.

\subsection{Snelson's X beam with three modules}

The Snelson's X beam shown in Figure 5 is made of three modules; its topology and geometry are described in [27]. The Snelson's elementary module has dimensions in $x$ and $y$ directions equal to $3000 \mathrm{~mm}$ and $2000 \mathrm{~mm}$, respectively. The tensegrity structure has 8 nodes and it is composed of 16 elements, 10 cables and 6 struts. From the analysis of the null-space of the equilibrium matrix $\mathbf{A}$, it results that its rank is equal to 13 , thus the tensegrity structure has 3 independent self-stress states. Moreover, the number of the

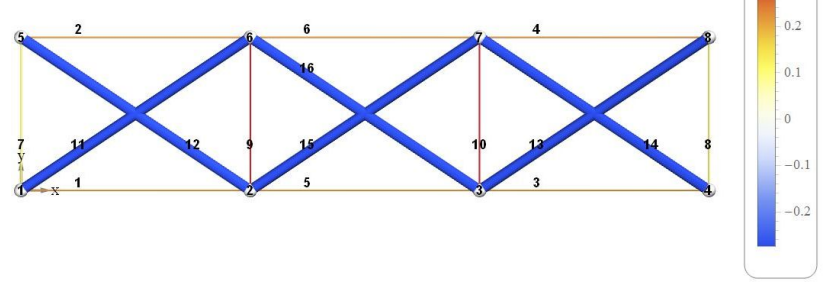

Figure 5: Snelson's X beam with three modules, perspective view. Thick cylinders represent the struts. Different colours have been assigned according to the value of the internal forces in the elements, which are labelled according to the connectivity matrix

infinitesimal mechanisms is equal to 0 , that is the Snelson's X tensegrity beam analyzed is kinematically determinate. The normalized feasible self-stress states evaluated by means of the algorithm presented in [32] are displayed in Figure 5.

In Table 3 are listed the assignments of the axial stiffness of the elements of the Snelson's X beam, whereas the related DSI values of the elements are shown in Figure 6.

In order to evaluate how the internal forces vary as the stiffness properties of a single group of the elements change, the case $n 1$ and the case $n 2$ differs only for the fact that in the case $\mathrm{n} 2$ the stiffness assigned to the cables 9-10 is greater than the stiffness assigned to the same elements in the case $n 1$. 
Table 3: Axial stiffness of the elements of the Snelson's X beam with three modules

\begin{tabular}{ccccccc}
\hline & $\mathbf{F}=\mathbf{I}$ & literature & $\mathrm{n} 1$ & $\mathrm{n} 2$ & $\mathrm{n} 3$ & \\
\hline Element & $E_{k} A_{k}(\mathrm{~N})$ & $E_{k} A_{k}(\mathrm{~N})$ & $E_{k} A_{k}(\mathrm{~N})$ & $E_{k} A_{k}(\mathrm{~N})$ & $E_{k} A_{k}(\mathrm{~N})$ & Element \\
\hline cables (1-4) & 3000 & $9.714 \cdot 10^{6}$ & $3.238 \cdot 10^{6}$ & $3.238 \cdot 10^{6}$ & $9.714 \cdot 10^{6}$ & cables (1-2) \\
cables (5-6) & 3000 & $6.48 \cdot 10^{5}$ & $3.238 \cdot 10^{6}$ & $3.238 \cdot 10^{6}$ & $3.238 \cdot 10^{6}$ & cables (3-6) \\
cables (7-8) & 2000 & $18.78 \cdot 10^{6}$ & $3.238 \cdot 10^{6}$ & $3.238 \cdot 10^{6}$ & $6.476 \cdot 10^{6}$ & cables (7-9) \\
cables (9-10) & 2000 & $24 \cdot 10^{6}$ & $3.238 \cdot 10^{6}$ & $16.19 \cdot 10^{6}$ & $12.952 \cdot 10^{6}$ & cable (10) \\
struts (11-14) & 3605.55 & $19.428 \cdot 10^{7}$ & $19.428 \cdot 10^{7}$ & $19.428 \cdot 10^{7}$ & $16.19 \cdot 10^{7}$ & struts (11-12) \\
struts (15-16) & 3605.55 & $19.428 \cdot 10^{7}$ & $19.428 \cdot 10^{7}$ & $19.428 \cdot 10^{7}$ & $19.428 \cdot 10^{7}$ & struts (13-16) \\
\hline
\end{tabular}

DSI of the elements of the Snelson's $\mathrm{X}$ beam -3 modules

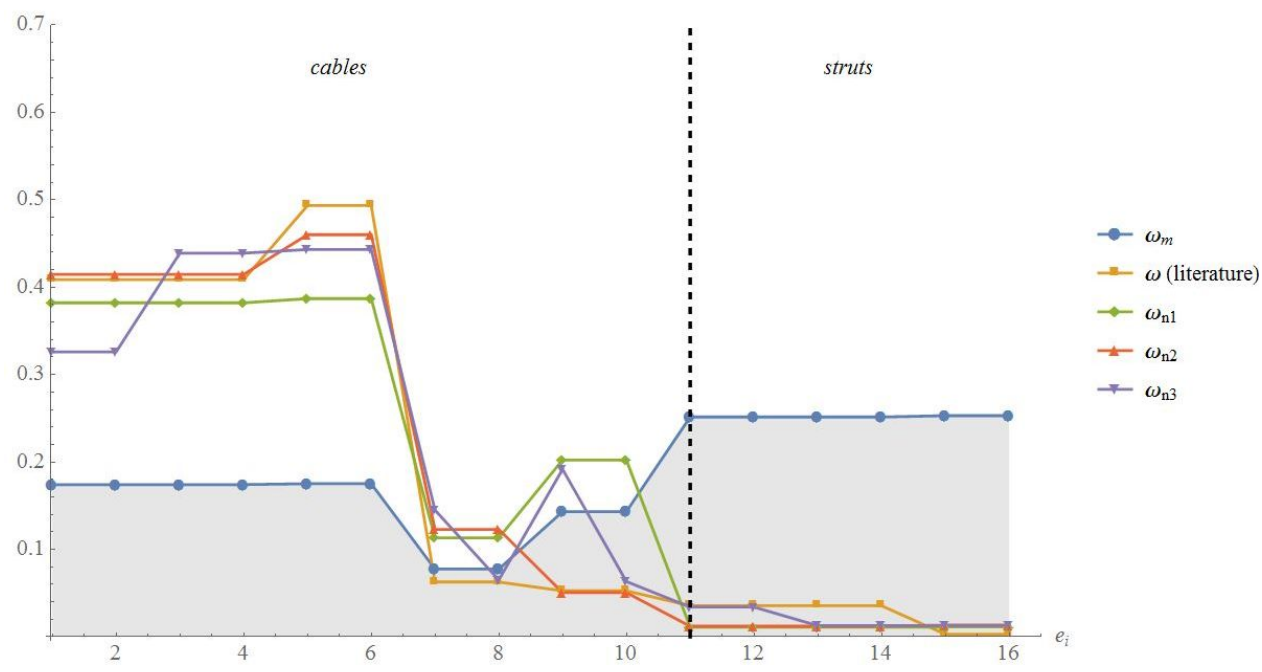

Figure 6: DSI of the elements of the Snelson's X beam with three modules for different assignments of the axial stiffness

Table 4: Axial stiffness of the elements of the Snelson's X beam with three modules which lead to the results obtained in the literature

\begin{tabular}{cccc}
\hline & exS1 & exS2 & exS3 \\
Element & $E_{k} A_{k}(\mathrm{~N})$ & $E_{k} A_{k}(\mathrm{~N})$ & $E_{k} A_{k}(\mathrm{~N})$ \\
\hline cables (1-4) & $1.44 \cdot 10^{6}$ & $3.238 \cdot 10^{4}$ & $19.428 \cdot 10^{6}$ \\
cables (5-6) & $4.69 \cdot 10^{5}$ & $8.42 \cdot 10^{3}$ & $5.11 \cdot 10^{6}$ \\
cables (7-8) & $64.76 \cdot 10^{6}$ & $16.19 \cdot 10^{6}$ & $16.19 \cdot 10^{6}$ \\
cables (9-10) & $64.76 \cdot 10^{6}$ & $16.19 \cdot 10^{6}$ & $3.238 \cdot 10^{6}$ \\
struts (11-14) & $25.9 \cdot 10^{7}$ & $97.14 \cdot 10^{6}$ & $19.428 \cdot 10^{7}$ \\
struts (15-16) & $25.9 \cdot 10^{7}$ & $12.95 \cdot 10^{7}$ & $97.14 \cdot 10^{6}$ \\
\hline
\end{tabular}

As well as for Quadruplex, by increasing the axial stiffness of cables 9-10, their DSI values decrease, whereas DSI values of the other elements increase.

It is worth to note that the feasible self-stress states obtained in literature, see [27], can be obtained for several distributions of the stiffness of the elements; three examples are listed in Table 4 (named exS1, exS2, exS3).
The analyses of the feasible self-stress states obtained by using the proposed method lead to the normalized internal forces in the elements shown in Figure 7.

It can be observed that for $\mathbf{F}=\mathbf{I}$, for the case named $\mathrm{n} 1$, as well as for the case $\mathrm{n} 2$, although the distribution of the axial stiffness of the elements is different, the elements of the tensegrity structure can be collected in the same groups according to the normalized internal forces. This happens because the matrix $\mathbf{\Omega}$ takes into account not only the stiffness symmetry but also the geometric symmetry properties of the structure.

By considering both the case $\mathrm{n} 1$ and $\mathrm{n} 2$, it can be seen that by increasing the axial stiffness of the cables 9-10, their internal forces increase. Simultaneously, the tensile internal forces in the cables 1-4 and in the cables 7-8, as well as the compressive internal forces in the struts 11-14 decrease. At the same time, the tensile internal forces in the cables $5-6$, as well as, the compressive internal forces in the struts 15-16 increase.

Such sensitivity analyses can be easily conducted by varying the Young's modulus and the cross-sectional area 


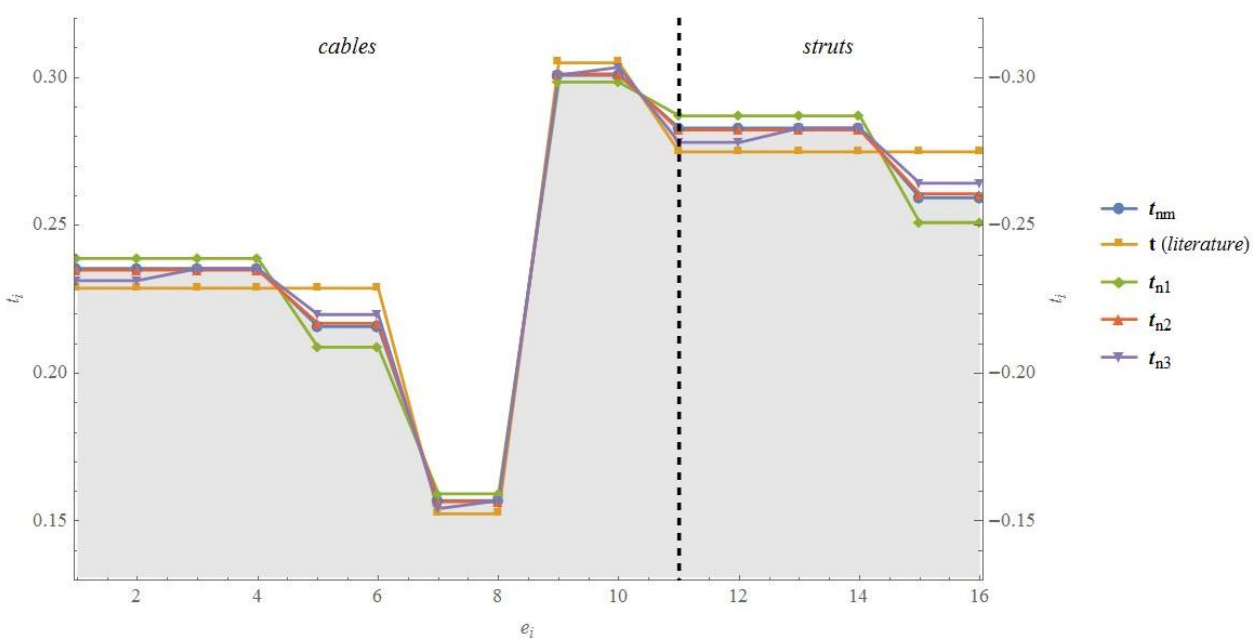

Figure 7: Internal forces in the elements of the tensegrity Snelson's X beam with three modules as the axial stiffness of the elements vary (note that the origin of the vertical axes is 0.13 )

Table 5: Force densities of the elements of the Snelson's X beam with three modules, normalized with respect to the force density of the first group

\begin{tabular}{ccccccc}
\hline & $\mathbf{F}=\mathbf{I}$ & literature & $\mathrm{n} 1$ & $\mathrm{n} 2$ & $\mathrm{n} 3$ & \\
Element & $q_{k}$ & $q_{k}$ & $q_{k}$ & $q_{k}$ & $q_{k}$ & Element \\
\hline cables (1-4) & 1 & 1 & 1 & 1 & 1 & cables (1-2) \\
cables (5-6) & 0.92 & 1 & 0.87 & 0.92 & 1.02 & cables (3-4) \\
cables (7-8) & 1 & 1 & 1 & 1 & 0.95 & cables (5-6) \\
cables (9-10) & 1.92 & 2 & 1.87 & 1.92 & 1 & cable (7) \\
struts (11-14) & -1 & -1 & -1 & -1 & 1.02 & cable (8) \\
struts (15-16) & -0.92 & -1 & -0.87 & -0.92 & 1.95 & cable (9) \\
& & & & & 1.97 & cable (10) \\
& & & & & -1.02 & struts $(11-12)$ \\
& & & & & -0.95 & struts $(13-14)$ \\
& & & & & &
\end{tabular}

Table 6: Norm of the unbalanced residual normalized internal forces vectors of the Snelson's X beam with three modules

\begin{tabular}{cccccc}
\hline & $\mathbf{t}_{\mathrm{nm}}$ & $\mathbf{t}$ (literature) & $\mathbf{t}_{\mathrm{n} 1}$ & $\mathbf{t}_{\mathrm{n} 2}$ & $\mathbf{t}_{\mathrm{n} 3}$ \\
\hline$\left[\left[\boldsymbol{\varepsilon}_{u}\right]\right]$ & $2.54 \cdot 10^{-16}$ & $4.74 \cdot 10^{-16}$ & $2.35 \cdot 10^{-16}$ & $2.15 \cdot 10^{-16}$ & $3.04 \cdot 10^{-16}$ \\
\hline
\end{tabular}

either of a unique element of the structure or of a single group of the elements.

Moreover, the same behaviour can be noted by examining the force densities of the elements, listed in Table 5, normalized respect to the force density of the first group. Such feasible force densities are in perfect agreement with the results obtained in [27] (refer to Table III in the reference).
The eigenvalues of the Force Density matrices $\mathbf{D}_{s}$, calculated by using the Eq. (25), are shown in Figure 8. It can be observed that these matrices are semi-positive definite and their rank deficiencies are equal to 5 . However, such Snelson's X beam has a degenerate geometry in a threedimensional space, thus, it is not super-stable.

The norms of the unbalanced residual normalized internal forces vectors [ $\left.\left[\boldsymbol{\varepsilon}_{u}\right]\right]$ are shown in Table 6 , and it can be 


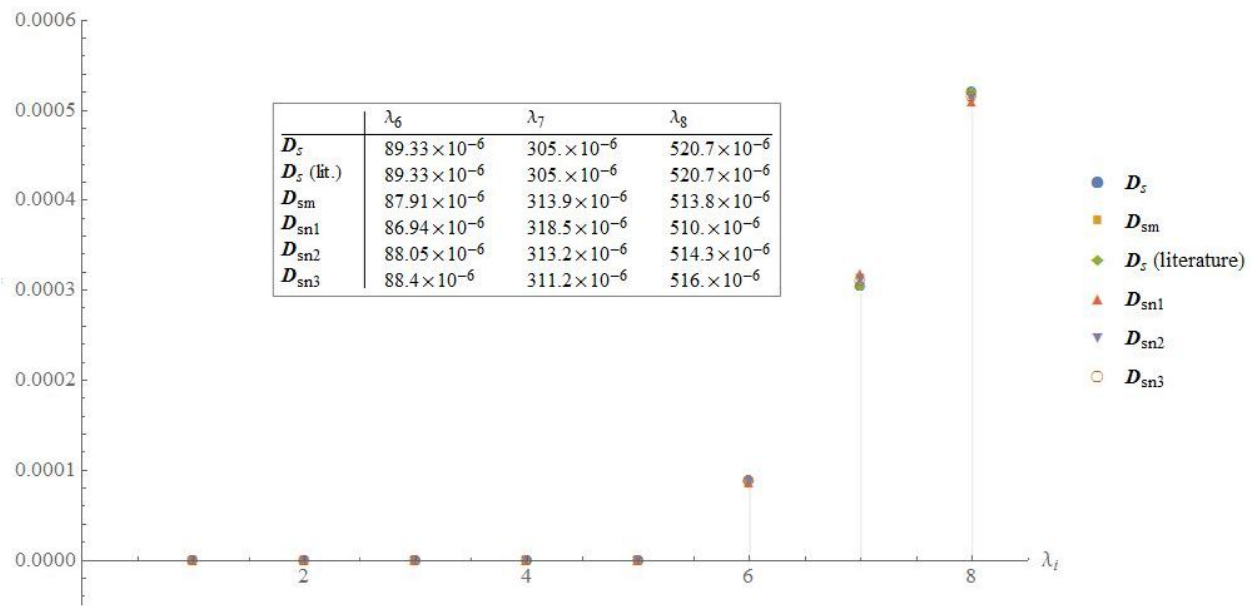

Figure 8: Eigenvalues of the Force Density matrices of the tensegrity Snelson's X beam with three modules

observed that such values are extremely close to 0 , which demonstrates the accuracy of the proposed method.

\subsection{Octahedral cell}

The Octahedral cell, shown in Figure 9, is made of 6 nodes and 15 elements, 12 cables and 3 struts. Its topology and geometry are illustrated in $[29,72]$. In particular, the length of the vertical strut (strut 15) is equal to $1000 \mathrm{~mm}$, whereas the lengths of the horizontal struts (struts 13-14) are equal to about $666.667 \mathrm{~mm}$. The feasible self-stress states presented in the literature [29, 72] are also shown in Figure 9.

The analysis of the equilibrium matrix A conducts to 3 independent self-stress modes, that is $s=3$, and 0 infinitesimal mechanisms, thus the Octahedral cell is a statically indeterminate and kinematically determinate tensegrity structure.

In Table 7 are listed the distribution of the axial stiffness of the elements of the Octahedral cell, whereas the related DSI values of the elements are illustrated in Figure 9-10. In particular, the case $\mathrm{n} 2$ differs from the case $\mathrm{n} 1$ only for the axial stiffness of the cables 1-4.
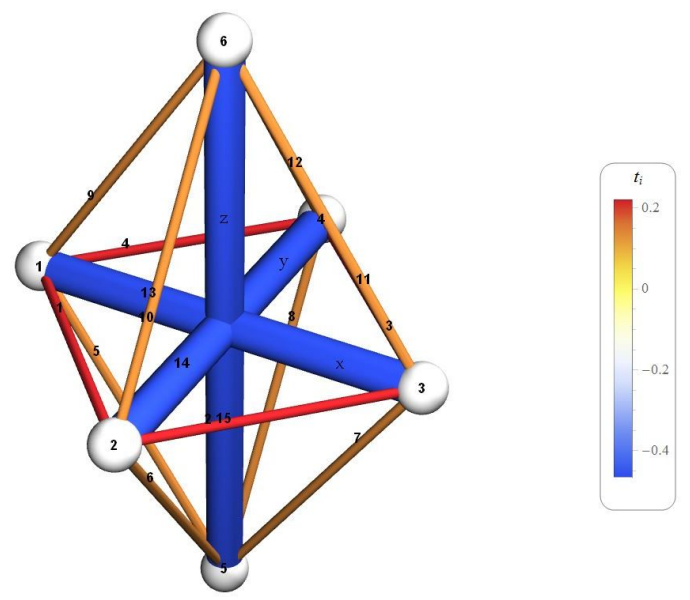

Figure 9: Octahedral cell, perspective view. Thick cylinders represent the struts. Different colours have been assigned according to the value of the internal forces in the elements, which are labelled according to the connectivity matrix

Also for the Octahedral cell, it can be observed that by increasing the axial stiffness of the cables 1-4 their DSI values decrease, whereas DSI values of the remaining elements increase. Moreover, also in this case, the feasible self-stress states reported in the literature can be obtained

Table 7: Axial stiffness of the elements of the Octahedral cell

\begin{tabular}{ccccccc}
\hline & $\mathbf{F}=\mathbf{I}$ & literature & $\mathrm{n} 1$ & $\mathrm{n} 2$ & $\mathrm{n} 3$ & \\
Element & $E_{k} A_{k}(\mathrm{~N})$ & $E_{k} A_{k}(\mathrm{~N})$ & $E_{k} A_{k}(\mathrm{~N})$ & $E_{k} A_{k}(\mathrm{~N})$ & $E_{k} A_{k}(\mathrm{~N})$ & Element \\
\hline cables (1-4) & 471.405 & $3.238 \cdot 10^{6}$ & $3.238 \cdot 10^{6}$ & $16.19 \cdot 10^{6}$ & $9.714 \cdot 10^{6}$ & cables $(1-2)$ \\
cables (5-12) & 600.925 & $19.99 \cdot 10^{6}$ & $3.238 \cdot 10^{6}$ & $3.238 \cdot 10^{6}$ & $3.238 \cdot 10^{6}$ & cables $(3-12)$ \\
struts (13-14) & 666.667 & $48.57 \cdot 10^{6}$ & $65.94 \cdot 10^{6}$ & $65.94 \cdot 10^{6}$ & $65.94 \cdot 10^{6}$ & strut (13) \\
strut (15) & 1000 & $48.57 \cdot 10^{6}$ & $65.94 \cdot 10^{6}$ & $65.94 \cdot 10^{6}$ & $32.97 \cdot 10^{6}$ & struts (14-15) \\
\hline
\end{tabular}


DSI of the elements of the Octahedral Cell

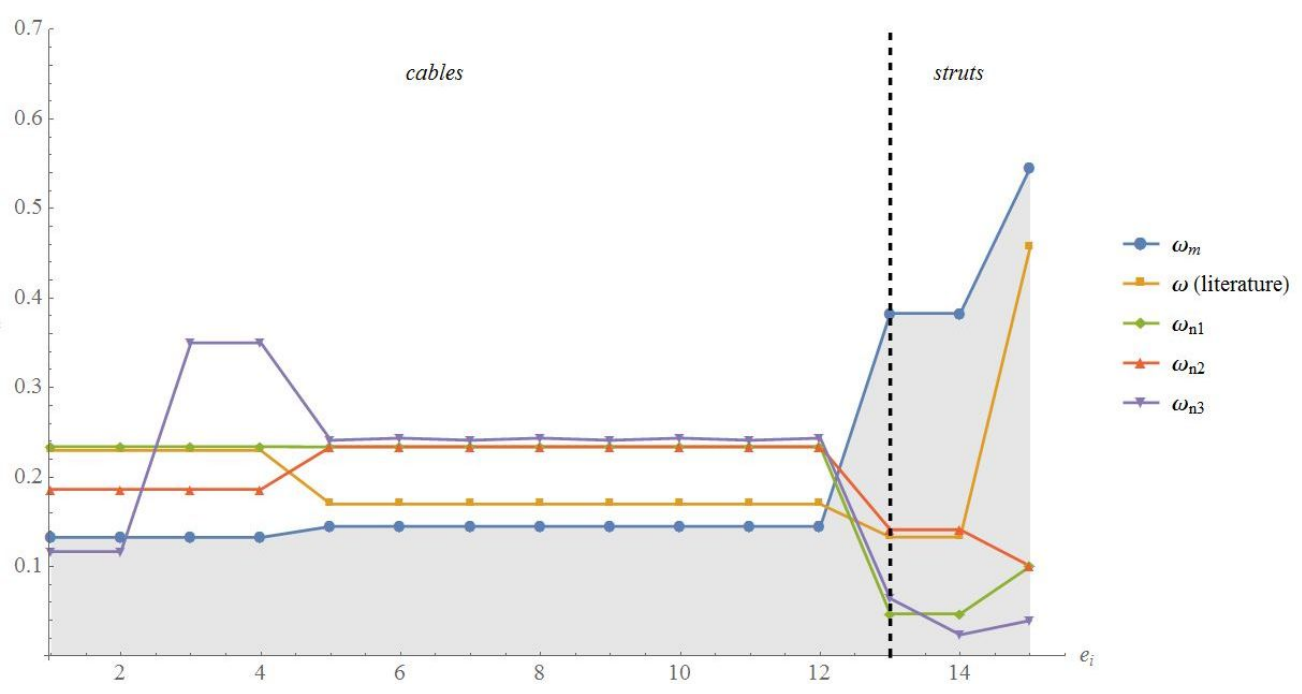

Figure 10: DSI of the elements of the Octahedral cell for different assignments of the axial stiffness

Internal forces in the elements of the Octahedral Cell

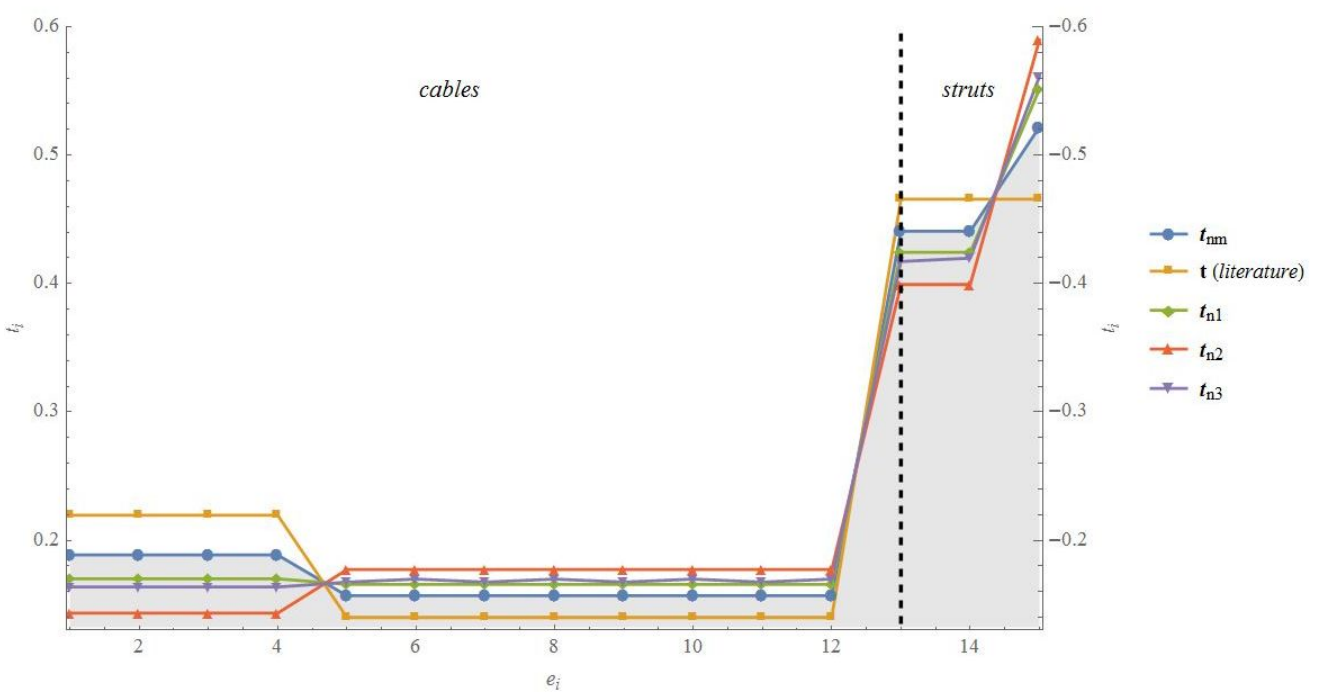

Figure 11: Internal forces in the elements of the Octahedral cell as the axial stiffness of the elements vary (note that the origin of the vertical axes is 0.13)

Table 8: Axial stiffness of the elements of the Octahedral cell which also lead to the results obtained in the literature

\begin{tabular}{cccc}
\hline & exO1 & exO2 & exO3 \\
Element & $E_{k} A_{k}(\mathrm{~N})$ & $E_{k} A_{k}(\mathrm{~N})$ & $E_{k} A_{k}(\mathrm{~N})$ \\
\hline cables (1-4) & $3.238 \cdot 10^{5}$ & $5.05 \cdot 10^{6}$ & $1.619 \cdot 10^{6}$ \\
cables (5-12) & $16.19 \cdot 10^{6}$ & $48.57 \cdot 10^{6}$ & $43.472 \cdot 10^{6}$ \\
struts (13-14) & $97.14 \cdot 10^{6}$ & $12.952 \cdot 10^{7}$ & $19.428 \cdot 10^{7}$ \\
strut (15) & $40.534 \cdot 10^{6}$ & $12.952 \cdot 10^{7}$ & $11.333 \cdot 10^{7}$ \\
\hline
\end{tabular}

for several assignments of the axial stiffness of the elements of the structure. In particular, in Table 8 are listed three possible assignments (termed exO1, exO2, exO3).

As it can be seen in Figure 11, for $\mathbf{F}$ equal to the identity matrix, as well as for the cases named $\mathrm{n} 1$ and $\mathrm{n} 2$, the elements of the Octahedral cell can be collected in the same groups according to the normalized internal forces. In particular, four groups can be identified: cables 1-4, cables 5-12, struts $13-14$ and strut 15 . Such a grouping scheme is consistent with both the geometrical symmetry and the stiffness symmetry of the structure. 
Table 9: Force densities of the elements of the Octahedral cell, normalized with respect to the force density of the first group

\begin{tabular}{ccccccc}
\hline & $\mathbf{F}=\mathbf{I}$ & literature & $\mathrm{n} 1$ & $\mathrm{n} 2$ & $\mathrm{n} 3$ & \\
Element & $q_{k}$ & $q_{k}$ & $q_{k}$ & $q_{k}$ & $q_{k}$ & Element \\
\hline cables (1-4) & 1 & 1 & 1 & 1 & 1 & cables (1-4) \\
cables (5-12) & 0.65 & 0.5 & 0.76 & 0.92 & 0.8 & cables $(5,7,9,11)$ \\
struts (13-14) & -1.65 & -1.5 & -1 & -1.76 & 0.81 & cables $(6,8,10,12)$ \\
strut (15) & -1.3 & -1 & -0.87 & -1.53 & -1.8 & strut (13) \\
& & & & & -1.81 & strut (14) \\
& & & & & -1.62 & strut (15) \\
\hline
\end{tabular}

Table 10: Norm of the unbalanced residual normalized internal forces vectors of the Octahedral cell

\begin{tabular}{cccccc}
\hline & $\mathbf{t}_{\mathrm{nm}}$ & $\mathbf{t}$ (literature) & $\mathbf{t}_{\mathrm{n} 1}$ & $\mathbf{t}_{\mathrm{n} 2}$ & $\mathbf{t}_{\mathrm{n} 3}$ \\
\hline$\left[\left[\boldsymbol{\varepsilon}_{u}\right]\right]$ & $6.38 \cdot 10^{-16}$ & $8.05 \cdot 10^{-16}$ & $6.23 \cdot 10^{-16}$ & $6.76 \cdot 10^{-16}$ & $6.27 \cdot 10^{-16}$ \\
\hline
\end{tabular}

Eigenvalues of the Force Density matrix of the Octahedral Cell

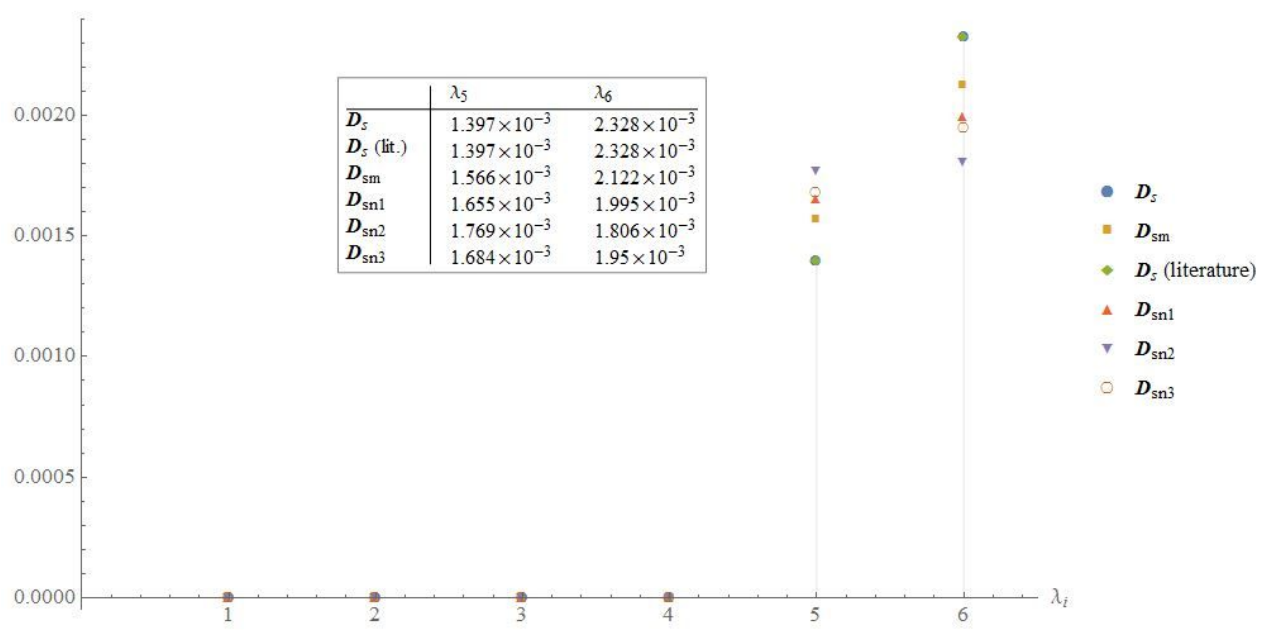

Figure 12: Eigenvalues of the Force Density matrices of the tensegrity Octahedral cell

Moreover, by comparing the case $\mathrm{n} 1$ with the case $\mathrm{n} 2$, it emerges that by increasing the axial stiffness of the cables 1-4 their tensile internal forces decrease, as well as the compressive internal forces in the horizontal struts 13-14 decrease. At the same time, the tensile internal forces in the cables 5-12 and the compressive internal force in the vertical strut 15 increases.

The normalized force densities of the elements of the Octahedral cell consistent with the different assignments of the axial stiffness of the members of the structure are listed in Table 9.

By using the Eq. (25) it is possible to determine the Force Density matrices for each of the feasible self-stress states; their eigenvalues are shown in Figure 12.

It can be noted that the Octahedral cell is a super-stable tensegrity structure; indeed, it has a non-degenerate geom- etry in the three-dimensional space, and its Force Density matrix is semi-positive definite with rank deficiency equal to 4. Such conditions occur for each of the axial stiffness assignments of the elements, thus for each feasible self-stress states obtained by using the proposed approach.

Finally, it is possible to calculate the norm of the unbalanced residual normalized internal forces vectors [ $\left.\left[\boldsymbol{\varepsilon}_{u}\right]\right]$, see Table 10, showing the accuracy of the proposed method.

\section{Discussion and conclusions}

A novel efficient method has been proposed for determining feasible self-stress states for tensegrity structures by 
investigating the Distributed Static Indeterminacy of the tensegrity structure.

The proposed methods have some advantages over the existing form-finding methods; in particular: (i) it allows for evaluating feasible self-stress states by performing only a unique Singular Value Decomposition of the equilibrium matrix A; (ii) it only requires, as preliminary information, the connectivity of the elements and their type, i.e. cable or struts, and the nodal coordinates; (iii) it is possible to obtain several feasible self-stress states as linear combinations of the independent self-stress modes according to the assignments of the axial stiffness of the elements.

This approach becomes particularly efficient for tensegrity structures with multiple self-stress states, as shown in the examined examples since it is not necessary to perform further SVD decompositions or to initialize grouping operations of the elements.

Indeed, such an approach consists of determining suitable linear combinations of the independent self-stress modes according to the axial stiffness of the elements. Thus, it overcomes difficulties arising with complicated optimization techniques, mixed-integer nonlinear programming strategies, spectral decompositions, stiffness matrix evaluations and numerical iterative procedures presented in the literature. This main feature reduces the time consuming of computational operations.

Moreover, it emerges that different feasible integral self-stress states can be easily obtained. In fact, feasible self-stress states consistent with the symmetry properties of the structures can be simply calculated by considering symmetric axial stiffness assignments to the elements of the tensegrity structure.

From the knowledge of the independent self-stress modes, and the evaluation of the Distributed Static Indeterminacy values related to the axial stiffness of each element, it is possible to address the self-stress identification of the tensegrity structures. Indeed, the proposed procedure bypass the element grouping operations needed in most of the state-of-the-art methods.

Furthermore, since DSI values are indicators that reflect the combined influence of the geometry, topology and axial stiffness of each element, different choices of element's stiffness lead to different feasible self-stress states. In particular, the load-bearing capacity of an element becomes lower as its DSI value increases. Thus, once evaluated the DSI of the elements of the structure, it is possible to calibrate each axial stiffness for achieving the desired mechanical behaviour of the entire structure.

The numerical analyses have shown that the norms of the unbalanced residual internal forces, evaluated for each case, are extremely close to zero, thus the accuracy of the proposed method has been proved.

Furthermore, the proposed approach allows to effectively determine the Force Density matrix of the structure, in order to evaluate the conditions of the super-stability for the tensegrity structure.

Moreover, the innovative method can be generalized by using a parametric description of the geometry of the structures in order to study how the internal forces in the elements vary as the geometrical parameters change, also for large-scale tensegrity structures.

Funding information: Authors state no funding involved.

Author contributions: All authors have accepted responsibility for the entire content of this manuscript and approved its submission.

Conflict of Interests: Authors state no conflict of interest.

\section{References}

[1] Fu F. Structural behavior and design methods of Tensegrity domes. J Construct Steel Res. 2005;61(1):23-5.

[2] Gómez-Jáuregui V, Arias R, Otero C, Manchado C. Novel Technique for Obtaining Double-Layer Tensegrity Grids. Int J Space Structures. 2012;27(2-3):155-66.

[3] Skelton RE, Fraternali F, Carpentieri G, Micheletti A. Minimum mass design of tensegrity bridges with parametric architecture and multiscale complexity. Mech Res Commun. 2014;58:124-32.

[4] Liapi K, Kim J. A Parametric Approach to the Design of Vaulted Tensegrity Networks. Int J Archit Comput. 2004;2(2):245-62.

[5] Cimmino MC, Miranda R, Sicignano E, Ferreira AJ, Skelton RE, Fraternali $F$. Composite solar façades and wind generators with tensegrity architecture. Compos, Part B Eng. 2017;115:275-81.

[6] Zolesi VS, Ganga PL, Scolamiero L, Micheletti A, Podio-Guidugli $P$, Tibert $G$, et al. On an innovative deployment concept for large space structures, In: 42nd Int. Conf. Environ. Syst., San Diego; 2012:1-14.

[7] Wendling S, Oddou C, Isabey D. Stiffening response of a cellular tensegrity model. J Theor Biol. 1999 Feb;196(3):309-25.

[8] Stamenović D, Fredberg JJ, Wang N, Butler JP, Ingber DE. A microstructural approach to cytoskeletal mechanics based on tensegrity. J Theor Biol. 1996 Jul;181(2):125-36.

[9] Djouadi S, Motro R, Pons JC, Crosnier B. Active Control of Tensegrity Systems. J Aerosp Eng. 1998;11(2):37-44.

[10] Liu K, Wu J, Paulino GH, Qi HJ. Programmable Deployment of Tensegrity Structures by Stimulus-Responsive Polymers. Sci Rep. 2017 Jun;7(1):3511.

[11] Yang S, Sultan C. Modeling of tensegrity-membrane systems. Int J Solids Struct. 2015;82:125-43.

[12] Nouri Rahmat Abadi B, Mehdi Shekarforoush SM, Mahzoon M, Farid M. Kinematic, Stiffness, and Dynamic Analyses of a Compliant Tensegrity Mechanism. J Mech Robot. 2014;6(4):041001. 
[13] Paul C, Valero-Cuevas FJ, Lipson H. Design and control of tensegrity robots for locomotion. IEEE Trans Robot. 2006;22(5):944-57.

[14] Sabelhaus A, Friesen J. SunSpiral V., Ji H., Hylton P., Madaan Y., et al., Mechanism Design and Simulation of the Ultra Spine, A Tensegrity Robot, In: Proceedings of ASME 2015 Int. Des. Eng. Tech. Conf. Comput. Inf. Eng. Conf. Boston; 2015:1-11.

[15] Chen LH, Kim K, Tang E, Li K, House R, Zhu EL, et al. Soft Spherical Tensegrity Robot Design Using Rod-Centered Actuation and Control. J Mech Robot. 2017;9(2):025001.

[16] Salahshoor H, Pal RK, Rimoli JJ. Material symmetry phase transitions in three-dimensional tensegrity metamaterials. J Mech Phys Solids. 2018;119:382-99.

[17] Rimoli JJ, Pal RK. Mechanical response of 3-dimensional tensegrity lattices. Compos, Part B Eng. 2017;115:30-42.

[18] Amendola A, Krushynska A, Daraio C, Pugno NM, Fraternali F. Tuning frequency band gaps of tensegrity metamaterials with local and global prestress. Int J Solids Struct. 2018;155:47-56.

[19] Fraddosio A, Pavone G, Piccioni MD. Minimal mass and selfstress analysis for innovative V-Expander tensegrity cells. Compos Struct. 2019;209:754-74.

[20] Fraddosio A, Marzano S, Pavone G, Piccioni MD. Morphology and self-stress design of V-Expander tensegrity cells. Compos, Part B Eng. 2017;115:102-16.

[21] Ferkiss V, Fuller RB, Applewhite EJ. Synergetics: Explorations in the Geometry of Thinking, Macmillan Pub Co. Technol Cult. 1976;17(1):104.

[22] Ashwear N, Eriksson A. Natural frequencies describe the prestress in tensegrity structures. Comput Struc. 2014;138:162-71.

[23] Oppenheim IJ, Williams WO. Geometric effects in an elastic tensegrity structure. J Elast. 2000;59(1/3):51-65.

[24] Tran HC, Lee J. Geometric and material nonlinear analysis of tensegrity structures. Acta Mech. Sin. Xuebao. 2011;27(6):93849.

[25] Zhang LY, Li Y, Cao YP, Feng XQ. Stiffness matrix based formfinding method of tensegrity structures. Eng Struct. 2014;58:3648.

[26] Gilewski W, Kłosowska J, Obara P. Form finding of tensegrity structures via Singular Value Decomposition of compatibility matrix, In: Proceedings of Adv. Mech. Theor. Comput. Interdiscip. Issues - 3rd Polish Congr. Mech. PCM 2015 21st Int. Conf. Comput. Methods Mech. C. Poland; 2015:191-96.

[27] Koohestani K. Automated element grouping and self-stress identification of tensegrities. Eng Comput. 2015;32(6):1643-60.

[28] Chen Y, Feng J, Ma R, Zhang Y. Efficient symmetry method for calculating integral prestress modes of statically indeterminate cable-strut structures. J Struct Eng. 2015;141(10):04014240.

[29] Tran HC, Lee J. Form-finding of tensegrity structures with multiple states of self-stress. Acta Mech. 2011;222(1-2):131-47.

[30] Sánchez R, Maurin B, Kazi-Aoual MN, Motro R. Selfstress States Identification and Localization in Modular Tensegrity Grids. Int J Space Structures. 2007;22(4):215-24.

[31] Quirant J, Kazi-Aoual MN, Motro R. Designing tensegrity systems: the case of a double layer grid. Eng Struct. 2003;25(9):1121-30.

[32] Tran HC, Lee J. Initial self-stress design of tensegrity grid structures. Comput Struc. 2010;88(9-10):558-66.

[33] Tibert AG, Pellegrino S. Review of Form-Finding Methods for Tensegrity Structures. Int J Space Structures. 2011;26(3):24155.

[34] Zhang JY, Ohsaki M. Adaptive force density method for formfinding problem of tensegrity structures. Int J Solids Struct.
2006;43(18-19):5658-73.

[35] Lee S, Lee J. A novel method for topology design of tensegrity structures. Compos Struct. 2016;152:11-9.

[36] Schek HJ. The force density method for form finding and computation of general networks. Comput Methods Appl Mech Eng. 1974;3(1):115-34.

[37] Xu X, Wang Y, Luo Y. An improved multi-objective topology optimization approach for tensegrity structures. Adv Struct Eng. 2018;21(1):59-70.

[38] Ehara S, Kanno Y. Topology design of tensegrity structures via mixed integer programming. Int J Solids Struct. 2010;47(5):5719.

[39] Zhang JY, Taguchi T. Form-Finding and Stability Analysis of Tensegrity Structures using Nonlinear Programming and Fictitious Material Properties. Int J Solids Struct. 2015;69-70:1-10.

[40] So AM, Ye Y. A semidefinite programming approach to tenseg. rity theory and realizability of graphs, In: Proceedings of seventeenth Annu. ACMSIAM Symp. Discret. algorithm SODA. Miami; 2006:766-75. https://doi.org/10.1145/1109557.1109641.

[41] Bel Hadj Ali N, Rhode-Barbarigos L, Smith IF. Analysis of clustered tensegrity structures using a modified dynamic relaxation algorithm. Int J Solids Struct. 2011;48(5):637-47.

[42] Fagerström G. Dynamic Relaxation of Tensegrity Structures, In: Proceedings of Between Man Mach. Proc. 14th Int. Conf. Comput. Archit. Des. Res. Asia, (22-25 April 2009, Yunlin, Taiwan) Taiwan; 2009:553-62.

[43] Pagitz M, Mirats Tur JM. Finite element based form-finding algorithm for tensegrity structures. Int J Solids Struct. 2009;46(17):3235-40.

[44] Klinka K, Arcaro V, Gasparini D. Form finding of tensegrity structures using finite elements and mathematical programming. J Mech Mater Struct. 2012;7(10):899-907.

[45] Chen Y, Feng J, Wu Y. Prestress stability of pin-jointed assemblies using ant colony systems. Mech Res Commun. 2012;41:30-6.

[46] Xu X, Luo Y. Form-finding of nonregular tensegrities using a genetic algorithm. Mech Res Commun. 2010;37(1):85-91.

[47] Feng $X$. The optimal initial self-stress design for tensegrity grid structures. Comput Struc. 2017;193:21-30.

[48] Linkwitz K, Schek HJ. Density Methods Applied to Form Finding of Initially Stressed Systems. Novel Approaches in Civil Engineering; 1971. pp. 341-50.

[49] Connelly R., Tensegrity structures. Why are they stable?, Rigidity theory Appl., 1998, 47-54.

[50] Xu X, Wang Y, Luo Y. Finding member connectivities and nodal positions of tensegrity structures based on force density method and mixed integer nonlinear programming. Eng Struct. 2018;166:240-50.

[51] Cai J, Wang X, Deng X, Feng J. Form-finding method for multi-mode tensegrity structures using extended force density method by grouping elements. Compos Struct. 2018;187:1-9.

[52] Cai J, Feng J. Form-finding of tensegrity structures using an optimization method. Eng Struct. 2015;104:126-32.

[53] Lee S, Lee J, Kang JW. Results of generalized equilibrium path from form-finding of tensegrity structure. Int J Steel Struct. 2017;17(3):1225-31.

[54] Gan BS, Zhang J, Nguyen DK, Nouchi E. Node-based genetic form-finding of irregular tensegrity structures. Comput Struc. 2015;159:61-73.

[55] Yuan XF, Ma S, Jiang SH. Form-finding of tensegrity structures based on the Levenberg-Marquardt method. Comput Struc. 
2017;192:171-80.

[56] Koohestani K. On the analytical form-finding of tensegrities. Compos Struct. 2017;166:114-9.

[57] Estrada GG, Bungartz HJ, Mohrdieck C. Numerical form-finding of tensegrity structures. Int J Solids Struct. 2006;43(22-23):685568.

[58] Calladine CR. Buckminster Fuller's “Tensegrity” structures and Clerk Maxwell's rules for the construction of stiff frames. Int J Solids Struct. 1978;14(2):161-72.

[59] Pellegrino S, Calladine CR. Matrix analysis of statically and kinematically indeterminate frameworks. Int J Solids Struct. 1986;22(4):409-28.

[60] Calladine CR, Pellegrino S. First-order infinitesimal mechanisms. Int J Solids Struct. 1991;27(4):505-15.

[61] Zhou J, Chen W, Zhao B, Qiu Z, Dong S. Distributed indeterminacy evaluation of cable-strut structures: formulations and applications. J. Zhejiang Univ. A. 2015;16(9):737-48.

[62] Yuan X, Chen L, Dong S. Prestress design of cable domes with new forms. Int J Solids Struct. 2007;44(9):2773-82.

[63] Zhou J, Chen W, Zhao B, Dong S. A feasible symmetric state of initial force design for cable-strut structures. Arch Appl Mech. 2017;87(8):1385-97.

[64] Zhang JY, Ohsaki M. Tensegrity Structures. Springer; 2015. https://doi.org/10.1007/978-4-431-54813-3.
[65] Chen Y, Sun Q, Feng J. Improved Form-Finding of Tensegrity Structures Using Blocks of Symmetry-Adapted Force Density Matrix. J Struct Eng. 2018;144(10):04018174.

[66] Zhang LY, Zhu SX, Li SX, Xu GK. Analytical form-finding of tensegrities using determinant of force-density matrix. Compos Struct. 2018;189:87-98.

[67] Tran HC, Lee J. Advanced form-finding of tensegrity structures. Comput Struc. 2010;88(3-4):237-46.

[68] Zhang JY, Ohsaki M. Stability conditions for tensegrity structures. Int J Solids Struct. 2007;44(11-12):3875-86.

[69] Lee S, Gan BS, Lee J. A fully automatic group selection for form-finding process of truncated tetrahedral tensegrity structures via a double-loop genetic algorithm. Compos, Part B Eng. 2016;106:308-15.

[70] Lee S, Lee J. Advanced automatic grouping for form-finding of tensegrity structures. Struct Multidiscipl Optim. 2017;55(3):95968.

[71] Kaveh A. Computational Structural Analysis and Finite Element Methods. Springer; 2014. https://doi.org/10.1007/978-3-31902964-1.

[72] Lee S, Lee J, Kang J. A Genetic Algorithm Based Form-finding of Tensegrity Structures with Multiple Self-stress States. J Asian Arch Build Eng. 2017;16:155-162. 


\section{A Appendix}

We can explicitly calculate $\mathbf{K} \mathbf{\Omega} \mathbf{F}$ in terms of the matrix $\mathbf{S}$. In particular, we have:

$$
\mathbf{K} \mathbf{\Omega} \mathbf{F}=\mathbf{K} \mathbf{F S}\left(\mathbf{S}^{\mathrm{T}} \mathbf{F S}\right)^{-1} \mathbf{S}^{\mathrm{T}} \mathbf{F}=\mathbf{S}\left(\mathbf{S}^{\mathrm{T}} \mathbf{F S}\right)^{-1} \mathbf{S}^{\mathrm{T}} \mathbf{F}
$$

Then we evaluate the transpose of the matrix $\mathbf{\Omega}$ :

$$
\mathbf{\Omega}^{\mathrm{T}}=\left(\mathbf{F S}\left(\mathbf{S}^{\mathrm{T}} \mathbf{F S}\right)^{-1} \mathbf{S}^{\mathrm{T}}\right)^{\mathrm{T}}
$$

Hence, from Eq. (A1) and Eq. (A2) it is clear that $\mathbf{K} \mathbf{\Omega F}$ is equal to $\mathbf{\Omega}^{\mathrm{T}}$.

$$
\begin{aligned}
& =\left(\left(\mathbf{S}^{\mathrm{T}} \mathbf{F S}\right)^{-1} \mathbf{S}^{\mathrm{T}}\right)^{\mathrm{T}}(\mathbf{F} \mathbf{S})^{\mathrm{T}}=\mathbf{S}\left(\left(\mathbf{S}^{\mathrm{T}} \mathbf{F S}\right)^{-1}\right)^{\mathrm{T}} \mathbf{S}^{\mathrm{T}} \mathbf{F} \\
& =\mathbf{S}\left(\left(\mathbf{S}^{\mathrm{T}} \mathbf{F S}\right)^{\mathrm{T}}\right)^{-1} \mathbf{S}^{\mathrm{T}} \mathbf{F}=\mathbf{S}\left((\mathbf{F S})^{\mathrm{T}} \mathbf{S}\right)^{-1} \mathbf{S}^{\mathrm{T}} \mathbf{F} \\
& =\mathbf{S}\left(\mathbf{S}^{\mathrm{T}} \mathbf{F S}\right)^{-1} \mathbf{S}^{\mathrm{T}} \mathbf{F} .
\end{aligned}
$$

